\title{
EXISTENCE OF LINEAR CONNECTIONS WITH RESPECT TO WHICH GIVEN TENSOR FIELDS ARE PARALLEL OR RECURRENT
}

\author{
YUNG-CHOW WONG
}

\section{Contents}

Introduction

1. Parallel or recurrent tensors on $M$.

1.1. Preliminaries.

1.2. Condition for existence of linear connections with respect to which given tensors are parallel or recurrent.

1.3. Some applications.

2. Conditions for a recurrent tensor to be almost-parallel.

2.1. The case $r \neq s$.

2.2. The case of recurrent tensor of type $(r, r)$.

2.3. Continuation. A main result.

2.4. A theorem on recurrent tensors of type $(r, r)$ which are not almost-parallel.

2.5. A sufficient condition for a recurrent tensor of type $(r, r)$ to be almost-parallel.

2.6. Some other conditions for a recurrent tensor of type $(r, r)$ to be almost parallel.

2.5. Some examples.

3. The matrix equation $\left(\otimes^{r} k\right) C\left(\otimes^{r} k^{-1}\right)=\sigma C$, and proof of Proposition 2.2.

3.1. Direct sums and direct products of matrices.

3.2. Proof of Proposition 2.2.

3.3. The $C^{*}$ 's for small values of $n$ and $r$.

4. Proof of Theorem 2.10.

4.1. Two lemmas.

4.2. The proof.

\section{Introduction}

Let $M$ be an $n$-dimensional $(n \geq 2)$ connected $C^{\infty}$-manifold with a linear connection. For simplicity, tensor fields on $M$ will simply be called tensors on $M$. A tensor $S$ on $M$ is said to be parallel if its covariant derivative is everywhere zero in $M$, i.e., if $\nabla S=0 . S$ is said to be recurrent if its covariant derivative is equal to the tensor product of a covector and $S$ itself, i.e., if $\nabla S=W \otimes S$, where $W$ is called the recurrence covector. A recurrent tensor $S$ on $M$ is said to be almost-parallel if there exists on $M$ some linear connection with respect

Received March 26, 1963. 
to which $S$ is parallel. In these definitions, we assume that neither the tensor $S$ nor the covector $W$ is everywhere zero in $M$, so that a tensor cannot be both recurrent and parallel with respect to the same linear connection.

Following a study by $\mathrm{H}$. S. Ruse of 3 - and 4-dimensional Riemannian manifolds whose curvature tensors are recurrent, A. G. Walker ([11]) defined recurrent tensors in $\boldsymbol{n}$-dimensional Riemannian manifolds and studied their relations with parallel fields of planes. These works of Ruse and Walker, though originally of a local nature, have led to developments in two directions: Linear connections with recurrent curvature (Wong [14]) and existence of linear connections with respect to which given fields of planes are parallel ${ }^{11}$. As recurrent tensors and parallel fields of planes are closely related concepts, it is natural to study the existence of linear connections with respect to which given tensors are parallel or recurrent. This problem has been on the author's mind since the main results in Wong [13] were obtained which seemed to provide a natural method to deal with the problem. But it was perhaps M. Kurita ([7]) who first studied the existence of linear connections with respect to which a given tensor is parallel.

In this paper, we first give an improvement of a result of Kurita's and a generalization to the case of recurrent tensors (Theorems 1.1-1.3). It follows from our results that with each parallel or recurrent tensor of type $(r, s)$ on $M$ there is associated a tensor of type $(r, s)$ over the $n$-dimensional (real) vector space $R^{n}$. This gives rise to the question: Under what conditions will a recurrent tensor be almost-parallel? It turns out that the answer is quite different according as the recurrent tensor is of type $(r, s), r \neq s$, or of type $(r, r)$. In fact, we prove that every recurrent tensor of type $(r, s), r \neq s$, is almost-parallel (Theorem 2.1) and that a recurrent tensor of type $(1,1)$ is almost-parallel iff it is nilpotent (Theorem 2.9). But the case of recurrent tensors of type $(r, r), r \geq 2$, is much more difficult. It leads to an equation which can be written symbolically as

$$
k \cdot C=\sigma C,
$$

where $C$ is the tensor of type $(r, r)$ over $R^{n}$ associated with the recurrent tensor, $k \cdot C$ denotes the action on $C$ induced from a non-singular linear trans-

1) For literature on those topics, see bibliographies in Willmore [12], pp. 276-278. 
formation $k$ in $R^{n}$, and $\sigma>0$ is a real number. A study of the consistency of this equation yields some necessary and some sufficient conditions for a recurrent tensor of type $(r, r)$ to be almost-parallel and the theorem that for a recurrent tensor of type $(r, r)$ which is not almost-parallel, the recurrence covector is locally a gradient (Theorem 2.4 ).

We shall give a fuller description of these results at the beginning of $\S 2$, but would like to mention here that our study of the equation $k \cdot C=\sigma C$ in the field of real numbers completely solves the problem of consistency of this equation in an algebraically closed field, giving a remarkable and perhaps important result (Proposition 3.1).

The author is grateful to Professor S. Ishihara for the many stimulating discussions on this subject, and to Professor H. C. Wang for supplying Lemma 1.3 and its proof. The author is also grateful to his young colleagues $\mathrm{Mr}$. C. $\mathrm{S}$. Hsü who read through critically a first draft of the manuscript and $\mathrm{Mr}$. K. Y. Lam who helped in certain computations and checking in $\S \S 3$ and 4 .

\section{Parallel or recurrent tensors on $M$}

The purpose of this section is to prove Theorems $1.1-1.3$ which give a necessary and sufficient condition for the existence on $M$ of a linear connection with respect to which given tensors are parallel or recurrent. Some applications are given.

\subsection{Preliminaries (cf. Wong [13]).}

We first give a few definitions and three lemmas which will be needed later. The indices $\alpha, \beta, \gamma, \varepsilon, i, j, k, \cdots$ all have the range $1, \cdots, n$. The ranges of other indices will be given as they appear. Summation over a repeated index is implied. Tensors, functions, and linear connections on $C^{\infty}$-manifolds are assumed to be $C^{\infty}$ unless stated otherwise.

Let $M$ be a connected $n$-dimensional $C^{\infty}$-manifold satisfying the second axiom of countability. A frame in $M$ is composed of a point $u \in M$ and an ordered set of $n$ linearly independent tangent vectors at $u$. To any frame $\left\{X_{\alpha}(u)\right\}$ in $M$ at $u$, there corresponds a unique dual coframe $\left\{\bar{X}^{\top}(u)\right\}$ in $M$ at $u$. In particular, when a fixed local coordirate system $\left(U, u^{i}\right)$ is given in $M$ with coordinate neighborhood $U$ and local coordinates $u^{i}$, there is attached to each point $u \in U$ the natural frame $\left\{\left(\partial / \partial u^{i}\right)_{u}\right\}$ and its dual coframe $\left\{\left(d u^{i}\right)_{u}\right\}$. 
Given a frame $z=\left\{X_{\alpha}(u)\right\}$ at $u \in M$, any tensor $S(u)$ at $u \in M$ can be expressed uniquely as

$$
S(u)=S_{\gamma}^{\alpha \beta \cdots}(z) X_{\alpha}(u) \otimes X_{\beta}(u) \otimes \bar{X}^{\Upsilon}(u) \otimes \cdots,
$$

where $\left\{\bar{X}^{\gamma}(u)\right\}$ is the dual coframe of $\left\{X_{\alpha}(u)\right\}$. We call the numbers $S_{\Upsilon}^{\alpha \beta \ldots}(z)$ the components of $S(u)$ relative to the frame $z=\left\{X_{\alpha}(u)\right\}$. In particular, if $S$ is a tensor defined on a coordinate neighborhood $U$, the components $S_{k \ldots}^{i j \ldots}$ of $S$ relative to the natural frame $\left\{\partial / \partial u^{i}\right\}$ are the usual components of $S$ in the local coordinate system $\left(U, u^{i}\right)$. It is easy to see that if $X_{\alpha}(u)=x_{\alpha}^{i} \partial / \partial u^{i}$, then

$$
S_{r}^{\alpha \beta \cdots}(z)=S_{k \cdots}^{i j \cdots} x_{i}^{z} x_{j}^{\beta} x_{r}^{k} \cdots,
$$

where $\left(x_{i}^{\alpha}\right)$ is the inverse of the matrix $\left(x_{\alpha}^{i}\right)$. Moreover, it follows at once from definition that the components of $S(u)$ relative to the frame $z(u)=\left\{X_{\alpha}(u)\right\}$ and those relative to the frame $z^{\prime}(u)=\left\{X_{\alpha}(u) g_{\alpha^{\prime}}^{\alpha}\right\}$ are related by

$$
S_{r}^{x^{\prime} \beta^{\prime} \cdots}\left(z^{\prime}(u)\right)=S_{r}^{\alpha \beta \cdots}(z(u)) \bar{g}_{\alpha}^{\alpha^{\prime}} \bar{g}_{\beta}^{\beta^{\prime}} g_{\Upsilon}^{\gamma} \cdots \cdots,
$$

where $\left(\bar{g}_{\alpha}^{\alpha^{\prime}}\right)$ is the inverse of the matrix $\left(g_{\alpha^{\prime}}^{\alpha}\right)$. For brevity, we shall write the above equation as

$$
\widetilde{S}\left(z^{\prime}(u)\right)=g^{-1} \cdot \widetilde{S}(z(u)) .
$$

Thus, $\widetilde{S}(z(u))$ denotes the set of components $S_{\Upsilon}^{\alpha \beta \cdots}(z(u))$ and $g^{-1}$ denotes an operator whose action on $\widetilde{S}(z(u))$ is as defined.

Let $B$ be the total space of the frame bundle $\pi: B \rightarrow M$ over $M$. A point $z$ in $B$ is a frame $\left\{X_{\alpha}(u)\right\}$ in $M$ at the point $u=\pi z \in M$. For any tensor $S$ on $M$, the components $S_{\mathrm{r}}^{\alpha \beta \cdots} \ldots(z)$ of $S(\pi z)$ relative to the frame $z$ define a set of functions $S_{r}^{\alpha \beta \cdots}$ on $B$, which in Wong [13], $\S 2.1$, were called the functions corresponding to the tensor $S$ on $M$.

Now assume that a linear connection has been given on $M$. If $B\left[z_{0}\right]$ is the submanifold of $B$, consisting of all the points which can be joined to a given point $z_{0}$ in $B$ by (sectionally $C^{\infty}$ ) horizontal curves, then we have (Wong [13], (3.15) and (3.9)) :

Lemma 1.1. On $M$ with a linear connection, a tensor $S$ is parallel iff the restrictions of its corresponding funtions on $B$ to any $B\left[z_{0}\right]$ are all constant, but not all zero.

LEMмA 1.2. On $M$ with a linear connection, a tensor $S$ is recurrent iff the 
restrictions of its corresponding functions on $B$ to any $B\left[z_{0}\right]$ have no common zero and are proportional to a fixed set of constants.

It follows at once from these that a parallel or recurrent tensor on $M$ is nowhere zero.

The following lemma and its proof were kindly supplied by Professor $\mathrm{H}$. C. Wang.

Lemma 1.3. Let $(u, y)=\left(u^{1}, \cdots, u^{n}, y^{1}, \cdots, y^{m}\right)$ be coordinates in the Euclidean $(n+m)$-space $R^{n+m}$ and $F_{\xi}(u, y), 1 \leq \xi \leq p, p C^{\infty}$-functions in $(u, y)$. Suppose that, for every fixed $u$, the set of points $(u, y)$ satisfying the equations $F_{\xi}(u, y)=0$ is a $C^{\infty}$ closed submanifold (of $R^{n+m}$ ) of dimension $q \leq m$, and $q$ is independent of $u$. Then the set $B^{\prime}$ of points $(u, y)$ satisfying the equations $F_{\xi}(u, y)=0$ is a $C^{\infty}$ closed submanifold of $R^{n+m}$ of dimension $n+q$.

Proof. Let $\left(u_{0}, y_{0}\right)$ be any point of $B^{\prime}$. It follows from assumption that the $p \times m$ matrix

$$
\left[\frac{\partial F_{\xi}}{\partial y^{\eta}}\right]_{\left(u_{0}, y_{0}\right)} \quad(1 \leq \xi \leq p, 1 \leq \eta \leq m)
$$

is of rank $t=m-q$. Therefore, after rearranging the $F_{\xi}$ and the $y^{\eta}$ if necessary, we may assume that the determinant of order $t$ lying at the upper left corner of the above matrix is not zero. Then by the implicit function theorem, we can solve the equations

$$
F_{1}(u, y)=0, \cdots, F_{t}(u, y)=0
$$

for $y^{1}, \cdots, y^{t}$; more precisely, there exist $t C^{\infty}$-functions

$$
f_{1}(u, \tilde{y}), \cdots, f_{t}(u, \tilde{y})
$$

in the variables $(u, \tilde{y})=\left(u^{1}, \cdots, u^{n}, y^{t+1}, \cdots, y^{m}\right)$ defined on a neighborhood of $\left(u_{0}, \tilde{y}_{0}\right)$ such that, on a suitable neighborhood $V_{0}$ of $\left(u_{0}, y_{0}\right)$, the system of equations (1.1) is equivalent to the system of equations

$$
y^{1}=f_{1}(u, \tilde{y}), \cdots, y^{t}=f_{t}(u, \tilde{y}) .
$$

Therefore, on $V_{0}$, the solutions of $F_{\xi}(u, y)=0$ are the same as those of

$$
\left\{\begin{array}{c}
y^{1}=y_{1}(u, \tilde{y}), \cdots, y^{t}=f_{t}(u, \tilde{y}), \\
F_{t+1}\left(u, f_{1}(u, \tilde{y}), \cdots, f_{t}(u, \tilde{y}), \tilde{y}\right)=0, \\
\cdot \cdot \cdot \cdot \cdot \cdot \cdot \cdot \\
F_{p}\left(u, f_{1}(u, \tilde{y}), \cdots, f_{t}(u, \tilde{y}), \tilde{y}\right)=0 .
\end{array}\right.
$$


But from the assumption that, for every fixed $u$, the solutions of $F_{\xi}(u, y)=0$ form a $C^{\infty}$ closed submanifold of dimension $q=m-t$, it follows that the last $p-t$ equations in (1.3) must all be satisfied identically. Therefore, on the neighborhood $V_{0}$ of $\left(u_{0}, y_{0}\right)$, the system of equations $F_{5}(u, y)=0$ is equivalent to (1.2); in other words, on the neighborhood $V_{0}$, the set $B^{\prime}$ is represented by the equations (1.2). Hence $B^{\prime}$ is a $C^{\infty}$ closed submanifold of $R^{n+m}$ of dimension $n+m-t=n+q$, as was to be proved.

\subsection{Condition for the existence of linear connections with respect to which given tensors are parallel or recurrent}

For convenience and clarity, we shall first prove the following two theorems dealing with a single tensor.

THEOREM 1.1. Let $S$ be any given tensor on $M$. Then there exists on $M a$ linear connection with respect to which $S$ is parallel iff we can assign to each point $u \in M$ a frame $z(u)$ such that the set $\widetilde{S}(z(u))$ of components of $S$ relative to $z(u)$ are not all zero and are independent of $u$.

THEOREM 1.2. Let $S$ be any given tensor on $M$. Then there exists on $M$ a linear connection with respect to which $S$ is recurrent iff we can assign to each point $u \in M$ a frame $z(u)$ such that the set $\widetilde{S}(z(u))$ of components of $S$ relative to $z(u)$ are not all zero and are proportional to a fixed set $\tilde{C}$ of constants (which are independent of $u$ ).

Remark. We note that in Theorems 1.1 and 1.2 and in Theorem 1.3 to be given later, the assignment of $z(u)$ to $u \in M$ is not required to be locally continuous, not to say locally $C^{\infty}$. This is an important fact which greatly facilitates the applications of our theorems.

Proof. Only the proof of Theorem 1.2 will be given, the proof of Theorem 1.1 being similar.

To prove the necessity of the condition, we use Lemma 1.2 and obtain

$$
S_{\Upsilon}^{\alpha \beta \cdots}(z)=\phi(z) C_{\Upsilon \cdots}^{\alpha \beta \cdots} \quad \text { on } B\left[z_{0}\right],
$$

where $\phi(z)$ is a $C^{\infty}$ and nowhere-zero function on $B\left[z_{0}\right]$, and $C_{\gamma \cdots}^{\alpha \beta \cdots}$ are constants, not all zero. For brevity, we shall write the above equation as

$$
\widetilde{S}(z)=\phi(z) \tilde{C} \quad \text { on } B\left[z_{0}\right] \text {. }
$$

Now for any point $u \in M$, let $u(\tau), 0 \leq \tau \leq$, be any (sectionally $C^{\infty}$ ) curve in $M$ 
joining the fixed point $u_{0}=\pi z_{0} \in M$ to the point $u$. If $z(\tau)$ is the lift of $u(\tau)$ in $B$ passing through $z_{0}$, then the end point of $z(\tau)$ belongs to $\pi^{-1}(u) \cap B\left[z_{0}\right]$. Thus, by (1.4); this point is a frame $z(u)$ in $M$ at $u$ such that

$$
\widetilde{S}(z(u))=\phi(z(u)) \widetilde{C} \equiv \rho(u) \widetilde{C},
$$

which proves the necessity of the condition in Theorem 1.2. We note here that since the $C^{\infty}$-function $\phi(z)$ is nowhere zero in $B\left[z_{0}\right]$, its value is of the same sign everywhere in $B\left[z_{0}\right]$. Consequently, though the function $\rho(u)$ is generally not continuous, its value is of the same sign for every point $u \in M$.

To prove the sufficiency of the condition in Theorem 1.2, we assume that a tensor $S$ on $M$ has the property that at each point $u \in M$, there exists a frame $z(u)$ relative to which the set $\widetilde{S}(z(u))$ of components of $S$ are not all zero and are proportional to a fixed set $\widetilde{C}$ of constants. Then $S$ has no zero in $M$, and consequently, its components relative to any frame $z$ are not all zero.

Denote by $H$ the set of those elements of the real general linear group $G L(n, R)$ that are characterized by the following property:

Let $G L(n, R)$ act on a fixed $n$-dimensional vector space $R^{n}$ by $\left\{\boldsymbol{e}_{\alpha}\right\} \rightarrow\left\{\boldsymbol{e}_{\alpha} g\right\}$ $\equiv\left\{e_{\alpha} g_{\alpha^{\prime}}^{\alpha}\right\}$, where $\left\{e_{\alpha}\right\}$ is a basis of $R^{n}$ and $g=\left(g_{\alpha^{\prime}}^{\alpha}\right) \in G L(n, R)$. Then $h=\left(h_{\alpha^{\prime}}^{\alpha}\right)$ $\in H$ if $H \subset G L(n, R)$ and if

$$
\begin{gathered}
h^{-1} \cdot \widetilde{C}=\psi(h) \widetilde{C}, \\
C_{\gamma}^{\alpha \beta \cdots} \bar{h}_{\alpha}^{\alpha^{\prime}} \bar{h}_{\beta}^{\beta^{\prime}} h_{\gamma}^{\gamma} \cdots=\phi(h) C_{\gamma \cdots \delta_{\alpha}^{\alpha \beta} \delta_{\beta}^{\beta^{\prime}} \delta_{\gamma}^{\gamma}, \cdots,}
\end{gathered}
$$

where $\delta_{\alpha}^{\alpha^{\prime}}$ is the Kronecker delta, $\left(\bar{h}_{\alpha}^{\alpha^{\prime}}\right)$ the inverse of the matrix $\left(h_{\gamma^{\prime}}^{\gamma}\right)$, and $\phi(h)$ an unspecified non-zero constant depending on $h$. It is easy to see that the set $H$ thus defined is a closed subgroup of $G L(n, R)$. But a closed subgroup of a Lie group is a Lie subgroup (see, for example, Chevalley [1], p. 135). Therefore, $H$ is a Lie group and a Lie subgroup of $G L(n, R)$.

Next consider the set $B_{H}$ of all those frames in $M$ such that the set $\bar{S}(z)$ of components of $S$ relative to each of these frames are proportional to the fixed set $\widetilde{C}$ of constants, i.e.,

$$
B_{H}=\{z \mid z \in B, \widetilde{S}(z)=: \ddot{\phi}(z) \widetilde{C}\},
$$

where $\widetilde{\phi}(z)$ is an unspecified function of $z$. By the assumption of the theorem, for every point $u \in M$, there exists some $z(u) \in B_{H}$. We now prove that 


$$
B_{H} \cap \pi^{-1}(u)=z(u) H \equiv\{z(u) h \mid h \in H\} .
$$

In fact, let $z^{\prime}(u)$ be any point in $B_{H} \cap \pi^{-1}(u)$. Then we have

$$
\widetilde{S}\left(z^{\prime}(u)\right)=\widetilde{\phi}\left(z^{\prime}(u)\right) \widetilde{C},
$$

and

$$
z^{\prime}(u)=z(u) g \quad \text { for some } g \in G L(n, R)
$$

From these and

$$
\tilde{S}(z(u) g)=g^{-1} \cdot \widetilde{S}(z(u)), \quad \widetilde{S}(z(u))=\tilde{\phi}(z(u)) \widetilde{C},
$$

it follows that

$$
\widetilde{\phi}(z(u)) g^{-1} \cdot \widetilde{C}=\widetilde{\phi}\left(z^{\prime}(u)\right) \widetilde{C} .
$$

Comparing this with the definition (1.6) of $H$, we see that $g \in H$, and this proves (1.8).

It is clear from (1.8) that under the action of $G L(n, R)$ on $B, H$ leaves $B_{H}$ invariant. Moreover, $H$ acts on $B_{H}$ without fixed point since this is true of the action of $G L(n, R)$ on $B$. Hence we may conclude from (1.8) that for eain point $u \in M, B_{H} \cap \pi^{-1}(u)$ is a $C^{\infty}$ closed submanifold of $B$ of the same dimension as $H$.

We now proceed to prove that $B_{H}$ is a $C^{\infty}$ closed submanifold of $B$. Since not all the constants $C_{r}^{\alpha \beta \cdots}$ are zero, there is at least one, say $C_{r_{0}^{0} \cdots}^{\alpha_{0} \beta_{0} \cdots}$, which is not zero. Then it is easy to see from definition (1.7) that

$$
B_{H}=\left\{z \mid z \in B, C_{r_{0} \cdots \cdots}^{\alpha_{0} \beta_{0} \cdots} S_{r \cdots}^{\alpha \beta \cdots}(z)=C_{r}^{\alpha \beta \cdots} S_{\Upsilon_{0}^{0} \cdots}^{\alpha_{0} \beta_{0} \cdots}(z)\right\} .
$$

Now in any coordinate neighborhood $\pi^{-1}(U)$ in $B$ where the local coordinates of a point $z$ are $(u, x) \equiv\left(u^{i}, x_{\alpha}^{i}\right)$, we have

$$
S_{r}^{\alpha \beta \cdots}(z)=S_{k \cdots}^{i j \cdots x_{i}^{\alpha} x_{j}^{\beta} x_{\top}^{k} \cdots} .
$$

Therefore, $B_{H} \cap \pi^{-1}(U)$ is the set of all points $(u, x)$ satisfying the equations

$$
F_{\Upsilon}^{\alpha \beta \cdots}(u, x) \equiv C_{\tau_{0} \cdots}^{\alpha_{0} \beta \cdots} S_{k \cdots}^{i j \cdots} x_{i}^{\alpha} x_{j}^{\beta} x_{\Upsilon}^{k} \cdots-C_{\Upsilon}^{\alpha \beta \cdots} \cdots S_{k \cdots}^{i j \cdots} x_{i}^{\alpha} x_{j}^{\alpha_{j}^{\beta}} x_{r_{0}}^{i} \cdots=0 .
$$

Since $S_{k \cdots}^{i j \cdots}$ are $C^{\infty}$ functions in $u$ and $\left(x_{i}^{\alpha}\right)$ is the inverse of the matrix $\left(x_{\alpha}^{i}\right)$, $F_{\gamma \ldots . .}^{\alpha \beta}(u, x)$ are $C^{\infty}$ functions in $(u, x)$. Moreover, for every fixed $u$, the set of points $(u, x)$ satisfying the equations $F_{r}^{2 \beta \cdots}(u, x)=0$ is $B_{H} \cap \pi^{-1}(u)$ which has just been shown to be a $C^{\infty}$ closed submanifold of $\pi^{-1}(U)$ of the same dimension as $H$. Thus, the conditions in Lemma 1.3 are satisfied and therefore, $B_{H} \cap \pi^{-1}(U)$ 
is a $C^{\infty}$ closed submanifold of $\pi^{-1}(U)$. From this it follows that $B_{H}$ is $a C^{\infty}$ closed submanifold of $B$, as was to be proved.

Summing up the above results, we may now conclude that in a natural manner, $B_{H} \rightarrow M$ is a principal fiber bundle with structure group $H$ which is $a$ subbundle of the frame bundle $B \rightarrow M$ with structure group $G L(n, R)$.

Connections are known to exist on any principal fiber bundle satisfying the second axiom of countability (see, for example, Nomizu [9], Chapter II, §9). Therefore, connections exist on $B_{H} \rightarrow M$. Furthermore, by means of the injection $B_{H} \rightarrow B$, any connection $\Gamma_{H}$ on $B_{H} \rightarrow M$ can be extended in a natural manner to a unique connection $\Gamma$ on $B \rightarrow M$, which is then a linear connction $\Gamma$ on $M$ (cf. Nomizu [9], Chapter II, §5). In fact, if the connection $\Gamma_{H}$ on $B_{H} \rightarrow M$ is defined by a field $Q_{H}$ of horizontal $n$-planes on $B_{H}$, then the field $Q$ of horizontal $n$-planes on $B$ defining the extended connection $\Gamma$ on $B \rightarrow M$ is obtained by extending $Q_{H}$ by the action of $G L(n, R)$ on $B$; more precisely, if $z$ is any point in $B$, we take any point $z_{1}$ in $B_{H}$ such that $z=z_{1} g$ for some $g \in G L(n, R)$, and define $Q_{z}$ as $\left(\mathrm{Q}_{H}\right)_{z_{1}} \cdot g$ which is easily seen to be independent of the choice of $z_{1}$.

We now show that, for any point $z_{0} \in B_{H} \subset B$, we have $B_{H}\left[z_{0}\right]=B\left[z_{0}\right]$. In fact, let $z_{1}$ be any point in $B_{H}\left[z_{0}\right]$. If $z_{H}(\tau), 0 \leq \tau \leq 1$, is any horizontal curve in $B_{H}$ joining $z_{0}$ to $z_{1}$, then since $z_{H}(\tau)$ is also a horizontal curve in $B, z_{1} \in B\left[z_{0}\right]$. Conversely, let $z_{2}$ be any point in $B\left[z_{0}\right]$ and $z(\tau), 0 \leq \tau \leq 1$, any horizontal curve in $B$ joining $z_{0}$ to $z_{2}$. Then $u(\tau)=\pi z(\tau)$ is a curve in $M$ joining $u_{0}=\pi z_{0}$ to $u_{2}$ $=\pi z_{2}$. If $z_{H}(\tau)$ is the lift of $u(\tau)$ in $B_{H}$ starting from $z_{0}$, then it is also a lift of $u(\tau)$ in $B$. But there is one and only one lift of $u(\tau)$ in $B$ passing through $z_{0}$. Therefore $z_{H}(\tau)=z(\tau)$, and $z_{2} \in z(\tau)=z_{H}(\tau) \subset B_{H}\left[z_{0}\right]$. Hence, $B_{H}\left[z_{0}\right]=B\left[z_{0}\right]$, as was to be proved.

From the way $B_{H}$ is constructed, the functions $S_{r}^{\alpha \beta \cdots}$ on $B_{H}$ have no common zero and are proportional to the fixed set of constants $C_{r}^{\alpha \beta \ldots}$. Therefore, $a$ fortiori, is this true of the restrictions of the functions $S_{\mathrm{r}}^{\alpha \beta \cdots}$ to $B_{H}\left[z_{0}\right]=B\left[z_{0}\right]$. Hence, by Lemma $1.2, S$ is recurrent with respect to the linear connection $\Gamma$ on $M$. This completes the proof of Theorem 1.2.

Theorems 1.1 and 1.2 can be extended to the following more general theorem.

Theorem 1.3. Let $S_{1}, \cdots, S_{p+q}$ be any $p+q$ given tensors on $M$. Then there exists on $M$ a linear connection with respect to which each of the tensors 
$S_{1}, \cdots, S_{p}$ is parallel and each of the tensors $S_{p+1}, \cdots, S_{p+q}$ is recurrent iff we can assign to each point $u \in M$ a frame $z(u)$ such that the components of $S_{1}, \cdots, S_{p}$ relative to $z(u)$ are not all zero and are independent of $u$, and the components of each of $S_{p+1}, \cdots, S_{p+q}$ relative to $z(u)$ are not all zero and are proportional to a fixed set of constants (which are independent of $u$ ), the factors of proportionality being generally different for different tensors $S_{p+1}, \cdots, S_{p+q}$.

proof. With slight modifications, the proof of Theorem 1.2 given above holds also for this theorem. We need only replace the single tensor $S$ by the tensors $S_{1}, \cdots, S_{p+q}$ and modify accordingly the definition of the subgroup $H$ of $G L(n, R)$ and that of the submanifold $B_{H}$ of $B$. More precisely, we let $\widetilde{C}_{\xi}(1 \leq \xi \leq p)$ and $\widetilde{C}_{\eta}(p+1 \leq \eta \leq p+q)$ be the sets of constants corresponding to the tensors $S_{\S}$ and $S_{\eta}$, respectively. Then we define $H$ to be the subgroup of $G L(n, R)$ consisting of those elements of $G L(n, R)$ such that

$$
h^{-1} \cdot \widetilde{C}_{\xi}=\widetilde{C}_{\xi} \quad(1 \leq \xi \leq p), \quad h^{-1} \cdot \widetilde{C}_{\eta}=\psi_{\eta}(h) \widetilde{C}_{\eta} \quad(p+1 \leq \eta \leq p+q),
$$

where $\psi_{\eta}(h)$ are unspecified and nowhere-zero functions of $h$. Correspondingly, we define $B_{H}$ to be the subset of $B$ consisting of those points $z$ of $B$ such that

$$
\widetilde{S}_{\xi}(z)=\widetilde{C}_{\xi}(1 \leq \xi \leq p), \widetilde{S}_{\eta}(z)=\widetilde{\phi}_{\eta}(z) \widetilde{C}_{\eta} \quad(p+1 \leq \eta \leq p+q),
$$

where $\widetilde{\phi}_{\eta}$ are unspecified functions of $z$. The rest of the proof is exactly the same as before.

Remark 1. Let $S$ be any tensor of type $(r, s)$ on $M$, and $S(z)$ the set of components of $S$ relative to the frame $z$. Then the conditions in Theorems 1.1 and 1.2 can be expressed respectively as

$$
\widetilde{S}(z(u))=\widetilde{C}, \quad \widetilde{S}(z(u))=\rho(u) \widetilde{C},
$$

where $\widetilde{C}$ denotes a set of constants, not all zero. Since $\widetilde{S}(z g)=g^{-1} \cdot \widetilde{S}(z)$ for any element $g$ of $G L(n, R)$, changing the family of frames $z(u)$ to $z(u) g$ changes the set $\widetilde{C}$ of constants to $g^{-1} \cdot \widetilde{C}$. Now if we regard $\widetilde{C}$ as the set of components of a tensor $C$ of type $(r, s)$ over the $n$-dimensional vector space $R^{n}$ relative to some basis $\left\{\boldsymbol{e}_{\alpha}\right\}$, then $g^{-1} \cdot \widetilde{C}$ is the set of components of the same tensor $C$ relative to the basis $\left\{e_{\alpha} g\right\}$. Therefore, with each parallel or recurrent tensor $S$ of type $(r, s)$ on $M$, there is associated a tensor $C$ of type $(r, s)$ over $R^{n}$.

Remark 2. Since local cross-sections of $B_{H} \rightarrow M$ exist, as is the case of all 
principal fiber bundles, we have the following interesting corollary to Theorems 1.1-1.3:

Let $S_{l}, \cdots, S_{p+q}$ be any $p+q$ tensors on $M$. Assume that we can assign (in any manner) to each point $u \in M$ a frame $z(u)$ such that the components of $S_{1}, \cdots, S_{p}$ relative to $z(u)$ are not all zero and are independent of $u$, and the components of each of $S_{p+1}, \cdots, S_{p+q}$ are not all zero and are proportional to a fixed set of constants (which are independent of $u$ ), the factors of proportionality being generally different for the different tensors $S_{p+1}, \cdots, S_{p+q}$. Then for each point $u \in M$ there is a neighborhood $U \ni u$ and a $C^{\infty}$ family of frames $z^{\prime}(u)$ in $U$ such that the components of the tensors $S_{1}, \cdots, S_{p+q}$ relative to $z^{\prime}(u)$ have the same properties as the components of these tensors relative to $z(u)$.

\section{3. Some applications}

A number of known theorems on existence of linear connections with specified properties are direct consequences of Theorem 1.3 and certain algebraic facts. For example, we can easily deduce that

If $S$ is any tensor of type $(0,2)$ on $M$ which (i) is symmetric and of constant rank or (ii) is skew-symmetric and of constant rank, then there exists on $M$ a linear connection with respect to which $S$ is parallel.

We now give another example to illustrate the arguments used. A complete system $D_{1}, \cdots, D_{p}$ of $p$ distributions on $M$ is such that at every point $u \in M$ the tangent space to $M$ at $u$ is the direct sum of $D_{1}(u), \ldots, D_{p}(u)$. With such a complete system of distributions there is associated a system of $p$ projection tensors $P_{1}, \cdots, P_{p}$ (of type $(1,1)$ ) which satisfy the conditions

$$
\Sigma_{\mu} P_{\mu}=E, P_{\mu}^{2}=P_{\mu}, P_{\mu} P_{\nu}=0, \quad(1 \leq \mu, \nu \leq p ; \mu \neq \nu)
$$

where $E$ is the unit tensor of type $(1,1)$. It is easy to show that if a linear connection has been given on $M$, a necessary and sufficient condition for each distribution $D_{\mu}$ to be parallel is that each tensor $P_{\mu}$ is parallel (see, for example, Fukami [4], p. 431).

On the other hand, we know from algebra (see, for example, Jacobson [6] p. 62) that, in the real field, if $P_{\mu}(1 \leq \mu \leq p)$ are any $p$ matrices of order $n$ satisfying (1.9), then there exists a non-singular matrix $F$ such that, for $\mu=1$, $\cdots, p$, 


$$
F P_{\mu} F^{-1}=\operatorname{diag}[0, \cdots, 0, \overbrace{1, \cdots, 1}^{n_{\mu}}, 0, \cdots, 0] \text {, }
$$

where $n_{\mu}$ is the rank of $P_{\mu}$, and the number of the first set of zeros in the diagonal is $n_{1}+\cdots+n_{\mu-1}$.

This fact implies, in our notation and terminology, that at each point $u \in M$, there exists a frame $z(u)$ relative to which the components of the tensors $P_{\mu}(z(u))$ are all independent of $u$. Thus, by Theorem 1.3 , there exists on $M$ a linear connection with respect to which each of the tensors $P_{\mu}$ is parallel. This proves the well-known theorem that

Given any complete system of distributions on $M$, there exists a linear connection with respect to which each of the distributions is parallel.

\section{Condition for a recurrent tensor to be almost-parallel}

The results in Theorems 1.1 and 1.2 give rise naturally to the question: Given on $M$ a tensor $S$ which is recurrent with respect to some given linear connection on $M$, when does there exist on $M$ a linear connection with respect to which $S$ is paralllel? In other words, when is a given rccurrent tensor almost-parallel? Here we recall from definitions (cf. Introduction) that a recurrent tensor is never a parallel tensor with respect to the same linear connection.

The answer to this question is completely different according as the recurrent tensor is of type $(r, s), r \neq s$, or of type $(r, r)$. The case of recurrent tensors of type $(r, s), r \neq s$, or of type $(1,1)$ can be dealt with easily (Theorems 2.1 and 2.8). But the case of recurrent tensors of type $(r, r), r \geq 2$, is much more difficult. It leads us to the equation

$$
k \cdot \widetilde{C}=\sigma \widetilde{C} \text {, i.e. } k_{\alpha_{1}}^{\gamma_{1}} \cdots k_{\alpha r}^{\gamma_{r}} C_{\beta_{1} \cdots \beta r}^{\alpha_{1} \cdots \alpha_{r}} \bar{k}_{\varepsilon_{1}}^{\beta_{1}} \cdots \bar{k}_{\varepsilon_{r}}^{\beta_{r}}=\sigma C_{\varepsilon_{1} \cdots \varepsilon_{r}}^{\gamma_{1} \ldots \gamma_{r}}
$$

where $\widetilde{C}$ denotes the set of constants $C_{\beta_{1} \ldots \beta r}^{\alpha_{1} \ldots \alpha_{r}}$ associated with the recurrent tensor, $\sigma>0$ is a parameter, $k=\left(k_{\alpha}^{\top}\right)$ is an element of $G L(n, R)$, and $\left(\bar{k}_{\varepsilon}^{\beta}\right)=k^{-1}$ (Theorem 2.2).

For given $\check{C}$ and $\sigma>0$, we say that $\widetilde{C}$ is consistent with $\sigma$ if there exists an element $k \in G L(n, R)$ such that $k \cdot \widetilde{C}=\sigma \widetilde{C}$. It is easy to see that the consistency of $\widetilde{C}$ with $\sigma$ is actually a property of the tensor $C$ of type $(r, r)$ over $R^{n}$ associated with the recurrent tensor (i.e. the tensor $C$ of type $(r, r)$ over 
$R^{n}$ with components $\widetilde{C}$ relative to some basis of $R^{n}$; see Remark 1 at the end of $\S 1.2)$. We prove that any given tensor $C$ of type $(r, r)$ over $R^{n}$ is consistent either with every $\sigma \in(0, \infty)$ or with only the elements of a totally disconnected subgroup of the multiplicative group $(0, \infty)$ (Proposition 2.1), and that a recurrent tensor $S$ of type $(r, r)$ on $M$ is or is not almost-parallel according as its associated tensor $C$ over $R^{n}$ is or is not consistent with every $\sigma \in(0, \infty)$ (Theorem 2.3). Although a more explicit criterion for deciding when a recurrent tensor of type $(\boldsymbol{r}, \boldsymbol{r})$ is almost-parallel has not yet been found, we are able to obtain several very interesting results. First we prove that for a recurrent tensor of type $(r, r)$ which is not almost-parallel, the recurrence covector is locally a gradient (Theorem 2.4). Next, we prove that if $C$ is consistent with some $\sigma_{0} \neq 1$ such that the characteristic roots of one of the $k \in G L(n, R)$ satisfying the equation $k \cdot \widehat{C}=\sigma_{0} \widehat{C}$ are all real, then $C$ is consistent with every $\sigma \in(0, \infty)$; moreover, we are able to construct all the tensors $C$ having this property (Proposition 2.2). This gives us a fairly general sufficient condition for a recurrent tensor of type $(r, r)$ to be almost-parallel (Theorems 2.5-2.6). In $\S 2.6$ we introduce the concept of nilpotence and complete nilpotence for tensors of type $(r, r)$, and obtain three necessary conditions for a recurrent tensor of type $(\boldsymbol{r}, \boldsymbol{r})$ to be almost-parallel (Theorems 2.7 and 2.10). We also prove that a recurrent tensor of type $(1,1)$ is almost-parallel iff it is nilpotent (Theorem 2.8). As examples, we show in $\$ 2.7$ how recurrent tensors which are or are not almost-parallel can be constructed on parallelisable $C^{\infty}$-manifolds.

Results on tensors over $R^{n}$ will be stated as propositions and those on recurrent tensors on $M$ will be stated as theorems.

\subsection{The case $r \neq s$}

We prove

THEOREM 2.1. Every recurrent tensor of type $(r, s), r \neq s$, is almost-parallel.

Proof. Assume that $S$ is a recurrent tensor of type $(r, s)$ on $M$. Then (see Theorem 1.2 and (1.5)), we may assign to each point $u$ in $M$ a frame $\boldsymbol{z}$ (u) such that

$$
\widetilde{S}(z(u))=\rho(u) \hat{C},
$$

where the function $\rho(u)$ is of the same sign for every point $u$. By replacing $\widetilde{C}$ by $-\widetilde{C}$ if necessary, we may assume that $\rho(u)>0$. 
Now assume that $r \neq s$. Since $\rho(u)>0$, we can find a non-zero number $\sigma(u)$ such that $\sigma^{r-s}(u)=\rho(u)$. Let $g(u)=\left(\sigma(u) \delta_{\alpha^{\prime}}^{\alpha}\right) \in G L(n, R)$. Then we have

$$
\widetilde{S}(z(u) g(u))=\widetilde{C} .
$$

This shows that $S$ satisfies the condition in Theorem 1.1, and consequently, there exists on $M$ a linear connection with respect to which $S$ is parallel.

\section{2. The case of recurrent tensors of type $(r, r)$.}

The object of this paragraph and the next is to prove Theorem 2.3 which gives a necessary and sufficient condition for a recurrent tensor of type $(\boldsymbol{r}, \boldsymbol{r})$ to be almost-parallel.

Let $S$ be a tensor of type $(r, r)$ which, with respect to some linear connection on $M$, is recurrent. Then by (1.4) and (1.5), there exists, at each point $u \in M$, a frame $z(u)$ such that

$$
\widetilde{S}(z(u))=\rho(u) \widetilde{C},
$$

where $\widetilde{C}=\left(C_{\beta_{1} \ldots \beta_{r}}^{\alpha_{1} \ldots \alpha_{r}}\right)$ is a set of constants not all zero, and $\rho(u) \equiv \phi(z(u))$ is of the same sign for every $u \in M$. We can prove that in this case the frame $z(u)$ at each point $u \in M$ can be so chosen that the number $\rho(u)$ is not independent of $u$ (see Lemma 2.1 at the end of this paragraph).

Now assume that there exists on $M$ a linear connection with respect to which $S$ is parallel. Then at each point $u \in M$, there exists a frame $z^{\prime}(u)$ such that

$$
\widetilde{S}\left(z^{\prime}(u)\right)=\widetilde{D},
$$

where $\widetilde{D}=\left(D_{\beta^{\prime}}^{\alpha \prime_{1}^{\prime} \ldots \beta^{\prime} r}\right)$ is a set of constants, not all zero. But

$$
z^{\prime}(u)=z(u) g(u) \text { for some } g(u) \in G L(n, R),
$$

and

$$
\widetilde{S}\left(z^{\prime}(u)\right)=g(u)^{-1} \cdot \widetilde{S}(z(u)) .
$$

Therefore, it follows from (2.1) and (2.2) that

$$
\rho(u) g(u)^{-1} \cdot \widetilde{C}=\widetilde{D} .
$$

Let us fix a point $u_{1}$ in $M$. From equation (2.3) and the same equation for $u=u_{1}$ we deduce that

$$
k(u) \cdot \widetilde{C}=\sigma(u) \widetilde{C},
$$


where

$$
k(u) \equiv g(u) g\left(u_{1}\right)^{-1}, \sigma(u) \equiv \rho(u) / \rho\left(u_{1}\right)>0 \text { and } \neq 1 .
$$

That $\sigma(u)>0$ and $\neq 1$ follows from the fact that $\rho(u)$ is of the same sign for every point $u \in M$ and is not independent of $u$. Thus the assumption that there exists on $M$ a linear connection with respect to which the recurrent tensor $S$ is parallel implies that, for each point $u \in M$, there exists an element $k(u)$ of $G L(n, R)$ such that $(2.4)$ is satisfied.

Conversely, if this condition is satisfied, then it follows from (2.1) and (2.4) that

$$
\widetilde{S}(z(u) k(u))=\rho(u) k(u)^{-1} \cdot \widetilde{C}=\rho\left(u_{1}\right) \widetilde{C} .
$$

This means that, at each point $u \in M$, there exists a frame $z^{\prime}(u)=z(u) k(u)$ relative to which the components of $S$ are the constants $\rho\left(u_{1}\right) \widetilde{C}$.

We have therefore proved

THEOREM 2.2. Let $S$ be a recurrent tensor of type $(r, r)$ on $M$ so that, for each point $u \in M$, there is a frame $z(u)$ relative to which $\widetilde{S}(z(u))=\rho(u) \tilde{C}$. Then a necessary and sufficient condition for $S$ to be almost-parallel is that there exists, for each point $u \in M$, an element $k(u)$ of $G L(n, R)$ such that

$$
k(u) \cdot \widetilde{C}=\sigma(u) \widetilde{C},
$$

where $\sigma(u)=\rho(u) / \rho\left(u_{1}\right)$ and $u_{1}$ is some fixed point in $M$.

We end this paragraph by proving the following lemma which was used in the proof of Theorem 2.2 and will be used again in the next paragraph.

LEMMA 2.1. If $S$ is recurrent so that $\widetilde{S}(z)=\phi(z) \widetilde{C}$ on $B\left[z_{0}\right]$, the assignment $u \rightarrow z(u) \in B\left[z_{0}\right]$ can be so chosen that the function $\rho(u) \equiv \phi(z(u))$ on $M$ has the property that, for some fixed $u_{1} \in M$ and variable $u \in M$, the set of values $\sigma(u) \equiv \rho(u) / \rho\left(u_{1}\right)$ covers entirely some interval $\left[1, \sigma_{1}\right]$ where $\sigma_{1}>1$.

Proof. Since $S$ is recurrent but not parallel, it follows from Lemmas 1.1 and 1.2 that the $C^{\infty}$ function $\phi$ on $B\left[z_{0}\right]$ is non-constant. Let $z_{3} \in B\left[z_{0}\right]$ be a point such that $\phi\left(z_{3}\right) \neq \phi\left(z_{0}\right)$ and let $z(\tau)$ be a horizontal curve joining $z_{0}$ to $z_{3}$. Then $\phi(z(\tau))$ is continuous in $\tau$. Let $z_{1}=z\left(\tau_{1}\right)$ be the farthest point from $z_{0}$ in $z(\tau)$ such that $\phi\left(z_{1}\right)=\phi\left(z_{0}\right)$. Then since $z(\tau)$ is horizontal and $\phi(z(\tau))$ is continuous in $\tau$, there exists a point $z_{2}=z\left(\tau_{2}\right), \tau_{1}<\tau_{2}$, in $z(\tau)$ sufficiently near 
$z_{1}$ such that $\phi\left(z_{2}\right) \neq \phi\left(z_{1}\right)$ and the curve $u(\tau) \equiv \pi z(\tau), \tau_{0} \leq \tau \leq \tau_{2}$, has no selfintersection. Now let us choose $z(\tau), \tau_{1} \leq \tau \leq \tau_{2}$, as the frame in $M$ at $u(\tau)$, $\tau_{1} \leq \tau \leq \tau_{2}$. Then $\rho(u)=\phi(z(u))$ has the property that $\rho(u(\tau))$ is continuous in $\tau$ and $\rho\left(u_{2}\right) \neq \rho\left(\boldsymbol{u}_{1}\right)$. One of these values is larger, and there is no loss of generality in assuming that $\rho\left(u_{2}\right)>\rho\left(u_{1}\right)(>0)$. Then putting $\rho\left(u_{2}\right) / \rho\left(u_{1}\right)=\sigma_{1}$, we prove our lemma.

\section{3. Continuation. A main result}

Theorem 2.2 leads us to the study of the consistency of the equation

$$
k \cdot \widetilde{C}=\sigma \widetilde{C} \text {, i.e. } k_{\alpha_{1}}^{\gamma_{1}} \cdots k_{\alpha_{r}}^{\gamma_{r}} C_{\beta_{1} \cdots \beta r}^{\alpha_{1} \cdots \alpha_{r}} \bar{k}_{\varepsilon_{1}}^{\beta_{1}} \cdots \bar{k}_{\varepsilon_{r}}^{\beta r}=\sigma C_{\varepsilon_{1} \cdots \varepsilon_{r}}^{\gamma_{1} \cdots \gamma_{r}},
$$

where $\widetilde{C}$ is a set of components $C_{\beta_{1} \ldots \beta r}^{\alpha_{1} \ldots \alpha_{r}}$ of a tensor of type $(r, r)$ over $R^{n}, k$ is some element of $G L(n, R)$, and $\sigma$ is a positive number.

We denote by $K$ the set of all elements $k \in G L(n, R)$ such that for each $k \in K$ there exists some $\sigma \in(0, \infty)$ satisfying (2.6), and denote by $\Sigma$ the set of all $\sigma \in(0, \infty)$ such that for each $\sigma \in \Sigma$ there exists some $k \in G L(n, R)$ satisfying (2.6). The sets $K$ and $\Sigma$ are both non-empty because, for any $\widetilde{C}$, equation (2.6) is satisfied by $\sigma=1$ and $k=$ identity matrix.

We now prove two lemmas on $K$ and $\Sigma$.

Lemma 2.2. $K$ is a subgroup of $G L(n, R), \Sigma$ is a subgroup of the multiplicative group $(0, \infty)$, and the correspondence $k \rightarrow \sigma$ defines a homomorphism $K \rightarrow \Sigma$. Furthermore, if $\sigma \in \Sigma$, then $\sigma^{m}(m= \pm 1, \pm 2, \cdots)$ all belong to $\Sigma$.

Proof. The statements in the Lemma are easy consequences of the fact that $k_{1} \cdot \widetilde{C}=\sigma_{1} \widetilde{C}$ and $k_{2} \cdot \widetilde{C}=\sigma_{2} \widetilde{C}$ imply that

$$
k_{1}^{-1} \cdot \widetilde{C}=\sigma_{1}^{-1} \widetilde{C} \text { and }\left(k_{2} k_{1}\right) \cdot \widetilde{C}=\left(\sigma_{2} \sigma_{1}\right) \widetilde{C} .
$$

Lемма 2.3. The subgroup $\Sigma$ of the multiplicative group $(0, \infty)$ is either totally disconnected or identical with the multiplicative group $(0, \infty)$.

Proof. Let $\Sigma_{0}$ be the connected component of the neutral element 1 in $\Sigma$. If $\Sigma_{0}=\{1\}, \Sigma$ is totally disconnected. If $\Sigma_{0} \neq\{1\}$, then $\Sigma_{0}$ is a closed interval containing \{1\}. From this and the fact that $\sigma \in \Sigma$ implies $\sigma^{m} \in \Sigma$ for $m= \pm 1$, $\pm 2, \cdots$, it follows that $\Sigma=(0, \infty)$, as was to be proved.

It was pointed out in $\S 1.3$ that with a recurrent or parallel tensor $S$ on $M$, there is associated a tensor $C$ over $R^{n}$ whose components relative to some basis are the set $\tilde{C}$ of constants. Now let $C$ be a tensor of type $(r, r)$ over $R^{n}$ 
with components $\widetilde{C}$ relative to some basis in $R^{n}$. We say that $C$ is consistent with the real number $\sigma \in(0, \infty)$ if the equation $k \cdot \widetilde{C}=\sigma \widetilde{C}$ holds for some element $k$ of $G L(n, R)$. This definition is legitimate because $k \cdot \widetilde{C}=\sigma \widetilde{C}$ and $\widetilde{C}^{\prime}=$ $g^{-1} \cdot \widetilde{C}, g \in G L(n, R)$, imply $\left(g^{-1} k g\right) \cdot \widetilde{C}^{\prime}=\sigma \widetilde{C}^{\prime}$ so that the consistency of $C$ with $\sigma$ is independent of the choice of basis in $R^{n}$. Thus we may say that a tensor $C$ is consistent with the number $\sigma \in(0, \infty)$ if it can be transformed into the tensor $\sigma C$ by the transformation extended from some non-singular linear transformation in $R^{n}$. If $C$ is consistent with some $\sigma \in(0, \infty)$, any $k \in G L(n, R)$ that satisfies $k \cdot \widetilde{C}=\sigma \widetilde{C}$ is said to be corresponding to $\sigma$. It is seen from above that the characteristic roots of $k$ are also independent of the choice of basis in $R^{n}$. (On the other hand, a change of basis in $R^{n}$ changes the group $K$ to one of its conjugates.)

We now restate Lemma 2.3 as

Proposition 2.1. A tensor $C$ of type $(r, r)$ over $R^{n}$ is consistent either with arbitrary $\sigma \in(0, \infty)$ or only with the elements of a totally disconnected subgroup of the multiplicative group $(0, \infty)$.

As a direct consequence of Theorem 2.2, Lemma 2.1 and Proposition 2.1, we have the following main result of this paragraph:

Theorem 2.3. A recurrent tensor $S$ of type $(r, r)$ on $M$ is almost-parallel or not almost-parallel according as the tensor $C$ of type $(r, r)$ over $R^{n}$ associated with it is consistent with every $\sigma \in(0, \infty)$ or only with the elements of a totally disconnected subgroup of the multiplicative group $(0, \infty)$.

We shall make a few applications of this theorem.

2.4. A theorem on recurrent tensors of type $(r, r)$ which are not almostparallel.

The object of this paragraph is to prove the interesting result stated in Theorem 2.4 below. To simplify the notation, composite indices will be used in part of this paragraph and in $\$ 2.6$. The composite indices $A, B, I, J$ are respectively the sets of ordered indices $\left(\alpha_{1}, \cdots, \alpha_{r}\right),\left(\beta_{1}, \cdots, \beta_{r}\right),\left(i_{1}, \cdots, i_{r}\right)$, $\left(j_{1}, \cdots, j_{r}\right)$. Since each of the indices $\alpha$ 's $\beta$ 's, $i$ 's, $j$ 's has the range $1, \cdots, n$, each of the composite indices $A, B, I, J$ has the range of $n^{r}$ values $(1, \cdots, 1)$, $\cdots,(n, \cdots, n)$ which we assume to have been arranged in the lexicographical order. Moreover, $A=B$. means that $\alpha_{1}=\beta_{1}, \cdots, \alpha_{r}=\beta_{r}$. 
In the notation of $\S 1.1$, for any tensor $S$ of type $(r, r)$ on $M$, we denote by $S_{B}^{A}(z)=S_{\beta_{1} \cdots \beta r}^{\alpha_{1} \cdots \alpha_{r}}(z)$ the components of $S$ relative to the frame $z=\left\{X_{\alpha}\right\}$ and by $S_{J}^{l}=S_{j_{1} \ldots j_{r}}^{i_{1} \ldots i_{r}}$ and $x_{\alpha}^{i}$ the components of $S$ and $X_{\alpha}$ in some local coordinate system in $M$. If $\left(x_{i}^{\alpha}\right)$ is the inverse of the matrix $\left(x_{\alpha}^{i}\right), g=\left(g_{\alpha^{\prime}}^{\alpha}\right)$ any element of $G L(n, R)$, and $\left(\bar{g}_{\alpha}^{\alpha^{\prime}}\right)$ the inverse of the matrix $\left(g_{\alpha^{\prime}}^{\alpha}\right)$, then we have $(\S 1.1)$

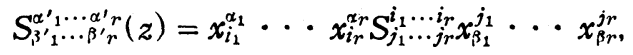

$$
\begin{aligned}
& S_{\beta^{\prime} \cdots \beta^{\prime} r}^{\alpha_{1} \cdots \alpha^{\prime} r}(z g)=\bar{g}_{\alpha_{1}}^{\alpha_{1}{ }^{\prime}} \cdots \bar{g}_{\alpha_{r}}^{\alpha^{\prime} r} S_{\beta_{1} \cdots \beta r}^{\alpha_{1} \cdots \alpha_{r}}(z) g_{\beta^{\prime}{ }_{1}}^{\beta_{1}} \cdots g_{\beta^{\prime} r}^{\beta_{r}} \text {. }
\end{aligned}
$$

Using composite indices, we write these equations as

$$
S_{B}^{A}=x_{I}^{A} S_{J}^{l} x_{B}^{\prime J}, \quad S_{B^{\prime}}^{A^{\prime}}(z g)=\bar{g}_{A}^{A \prime} S_{B}^{A}(z) g_{B^{\prime}}^{B} .
$$

We now prove

THEOREM 2.4. If a recurrent tensor of type $(r, r)$ on $M$ is not almostparallel, its recurrence covector is locally a gradient.

Proof. It follows from $\nabla S=W \otimes S$ that

$$
\nabla_{l} S_{J}^{L}=W_{l} S_{J}^{l}
$$

in every local coordinate system $\left(U, u^{i}\right)$ in $M$. The theorem asserts that if there exists no linear connection on $M$ with respect to which $S$ is parallel, then for each point $u_{1} \in M$ there exist a coordinate neighborhood $U \ni u_{1}$ and a $C^{\infty}$ function $\omega$ on $U$ such that $W_{l}=\partial \omega / \partial u^{l}$.

Since $S$ is recurrent, we have from Lemma 1.2 and (1.4) that

$$
\widetilde{S}(z)=\phi(z) \widetilde{C} \text { on } B\left[z_{0}\right],
$$

where $\phi$ is a $C^{\infty}$, non-constant, nowhere-zero function on $B\left[z_{0}\right]$, and $\widetilde{C}$ are $n^{2 r}$ constants, not all zero.

Let $z, z g$ be two points in $B\left[z_{0}\right]$, where $g \in G L(n, R)$. Then we have from (2.9) that

$$
\widetilde{S}(z g)=\phi(z g) \widetilde{C}, \quad \widetilde{S}(z)=\phi(z) \widetilde{C},
$$

which, together with $\widetilde{S}(z g)=g^{-1} \cdot \widetilde{S}(z)$, give

$$
g^{-1} \cdot \widetilde{C}=\{\phi(z g) / \phi(z)\} \widetilde{C} .
$$

Since $S$ is not almost-parallel, the tensor $C$ over $R^{n}$ with components $\widetilde{C}$ is consistent only with the elements of a totally disconnected subgroup $\Sigma$ of the multiplicative group $(0, \infty)$ (see Theorem 2.3). Thus it follows from (2.10) that 
if $z, z g$ are two points in $B\left[z_{0}\right]$,

$$
\phi(z g)=\sigma_{\lambda} \phi(z)
$$

where $\sigma_{\lambda}$ is some element of $\Sigma$.

Let $u_{1}$ be any point of $M$. Equation (2.11) shows that on each component (which is arcwise connected) of $\pi^{-1}\left(u_{1}\right) \cap B\left[z_{0}\right]$, the function $\phi$ is constant. Let us choose a sufficiently small coordinate neighborhood $U \ni \boldsymbol{u}_{1}$ in $M$ so that each component of $\pi^{-1}\left(u_{1}\right) \cap B\left[z_{0}\right]$ is contained in one and only one component of $\pi^{-1}(U) \cap B\left[z_{0}\right]$. Take any of the components, say $V$, of $\pi^{-1}(U) \cap B\left[z_{0}\right]$. Then $\phi$ is $C^{\infty}$ on $V$ and is constant on each fiber in $V$. Hence we can write the equation

$$
\widetilde{S}(z)=\phi(z) \widetilde{C} \text { on } V
$$

as

$$
\widetilde{S}(z)=\rho(u) \widetilde{C} \text { on } V,
$$

where $\rho(u) \equiv \phi(z(u))$, so that $\rho$ is a nowhere-zero, $C^{\infty}$ function on $U$.

Now we prove that on $U, W_{l}=\partial_{l} \log \rho$. Let $u(\tau)$ be any $C^{\infty}$-curve in $U$ passing through $u_{1}, z_{1}$ any point in $\pi^{-1}\left(u_{1}\right) \cap V$, and $z(\tau)$ the lift of $u(\tau)$ passing through $z_{1}$. Then $z(\tau)$ is a horizontal curve lying entirely in $V \subset B\left[z_{0}\right]$. Therefore, $z(\tau)=\left\{X_{\alpha}(u(\tau))\right\}$ is a $C^{\infty}$-field of parallel frames in $M$ along the curve $u(\tau)$, so that

$$
\frac{d u^{l}}{d \tau} \nabla_{l} x_{I}^{A}=0, \frac{d u^{l}}{d \tau} \nabla_{l} x_{B}^{J}=0
$$

Let us write 2.12$)$ in the form

$$
S_{B}^{A}(z)=\rho(u) C_{B}^{A} \quad \text { on } V,
$$

evaluate it along $z(\tau)$, and use $(2.7)_{1}$. Then we have

$$
\left(x_{I}^{A} S_{J}^{I} x_{B}^{J}\right)(u(\tau))=\rho(u(\tau)) C_{B}^{A} .
$$

If we differentiate this equation covariantly along the curve $\boldsymbol{u}(\tau)$ and make use of $(2.8)$ and (2.13), the result is

$$
x_{I}^{A}\left(\frac{d u^{l}}{d \tau} W_{l}\right) S_{, J}^{I} x_{B}^{J}=\left(\frac{d u^{l}}{d \tau} \partial \imath \rho\right) C_{B}^{A}
$$

which, together with $(2.14)$, gives 


$$
\left(\frac{d u^{l}}{d \tau} W_{l}\right) \rho C_{B}^{A}=\left(\frac{d u^{l}}{d \tau} \partial l \rho\right) C_{B}^{A}
$$

Since the constants $C_{B}^{A}$ are not all zero and the function $\rho$ is nowhere zero in $U$, it follows from the above equation that

$$
\frac{d u^{l}}{d \tau} W_{l}=\frac{d u^{l}}{d \tau} \partial_{l} \log \rho
$$

But the curve $u(\tau)$ through $u_{1}$ can be so chosen as to make its tangent vector $d u^{l} / d \tau$ at $u_{1}$ coincide with any given tangent vector in $M$ at $u_{1}$. Therefore, we have $W_{l}=\partial_{l} \log \rho$ at $u_{1} \in M$ and consequently everywhere in $U$. This completes the proof of Theorem 2.4.

Remark. It follows from (2.11) that if another component $V_{1}$ of $\pi^{-1}(U) \cap B\left[z_{0}\right]$ is used, the corresponding function $\rho_{1}$ is a constant multiple of the function $\rho$ corresponding to $V$. Therefore, we have

$$
W_{l}=\partial_{l} \log \rho=\partial_{l} \log \rho_{1}
$$

\subsection{A sufficient condition for a recurrent tensor of type $(r, r)$ to be almost- parallel}

Let us now return to Theorem 2.3 and recall that the problem of deciding when a recurrent tensor of type $(r, r)$ is almost-parallel is equivalent to the problem of consistency of a tensor of type $(r, r)$ over $R^{n}$ with a number $\sigma>0$. We shall prove later in $\S 3$ some remarkable results stated below in Proposition 2.2 which enable us to give a fairly general sufficient condition for a recurrent tensor of type $(r, r)$ to be almost-parallel (Theorem 2.5). In order to state our results in a convenient form we say that $C^{*}$ is a variable tensor (over $R^{n}$ ) if, relative to some basis in $R^{n}$, its non-zero components are independent variables. $A$ tensor $C$ is called a specialization of the variable tensor $C^{*}$ if its components $\widetilde{C}$ relative to some basis in $R^{n}$ are obtained from the components $\widetilde{C}^{*}$ of $C^{*}$ relative to the same basis by giving constant values to the independent variables contained in $\widetilde{C}^{*}$.

Proposition 2.2. If a tensor $C$ of type $(r, r)$ over $R^{n}$ is consistent with some $\sigma_{0}>0, \neq 1$, such that among the elements $k \in G L(n, R)$ corresponding to $\sigma_{0}$ there is one whose characteristic roots are all reai, then $C$ is consistent with any $\sigma>0$; and, for each $\sigma>0$, there exists a corresponding $k \in G L(n, R)$ whose characteristic roots are all real. 
Furthermore, a tensor $C$ of type $(r, r)$ over $R^{n}$ has the property described above iff it is a specialization of one of the variable tensors $C^{* \prime}$ s determined as follows:

(i) Let $\sigma>0$ be a parameter. Take any set (*) of $n-1$ independent and consistent equations of the form

$$
\sigma \lambda_{\beta_{1}} \cdots \lambda_{\beta_{r}}=\lambda_{\alpha_{1}} \cdots \lambda_{\alpha_{r}}
$$

in the $n$ unknowns $\lambda_{1}, \ldots, \lambda_{n}$.

(ii) Solve (*) for the ratios of $\lambda_{1}, \ldots, \lambda_{n}$, obtaining

$$
\lambda_{1}: \cdots: \lambda_{n}=\varepsilon_{1} \sigma^{q_{1}}: \cdots: \varepsilon_{n} \sigma^{q_{n}},
$$

where each $\varepsilon_{1}, \cdots, \varepsilon_{n}$ is +1 or -1 , and $q_{1}, \cdots, q_{n}$ are rational numbers.

(iii) Then the variable tensor $C^{*}$ corresponding to $(*)$ is obtained by putting

$$
C_{\varepsilon_{2} \cdots \varepsilon r}^{* r_{1} \ldots r_{r}}=0 \text { or an independent variable }
$$

according as the $\lambda_{1}, \cdots, \lambda_{n}$ given in (ii) satisfy

$$
\sigma \lambda_{\varepsilon_{1}} \cdots \lambda_{\varepsilon_{r}}-\lambda_{r_{1}} \cdots \lambda_{r r} \neq 0 \text { or }=0 .
$$

We note that since the number of such sets $(*)$ of equations of the form $\sigma \lambda_{\beta_{1}} \cdots \lambda_{\beta r}=\lambda_{\alpha_{1}} \cdots \lambda_{\alpha_{r}}$ is finite, there are only a finite number of such variable tensors $C^{*}$ s.

Thus, combining Theorem 2.3 with Proposition 2.2, we obtain the following theorem.

TheOREM 2.5. A recurrent tensor $S$ of type $(r, r)$ on $M$ is almost-parallel if the tensor $C$ of type $(r, r)$ over $R^{n}$ associated with it is consistent with some $\sigma_{0}>0, \neq 1$, and if corresponding to $\sigma_{0}$ there is $a k_{0} \in G L(n, R)$ whose characteristic roots are all real.

$A$ recurrent tensor of type $(r, r)$ on $M$ has the above property iff the tensor $C$ associated with it is a specialization of one of the variable tensors $C^{* \prime} s$ determined in Proposition 2.2.

For small values of $\boldsymbol{n}$ and $r$, the variable tensors $C^{* \text { 's }}$ described in Proposition 2.2. can be determined easily (\$3.3). For example, we have

TheOREM 2.6. A recurrent tensor of type $(2,2)$ on a 2-dimensional $C^{\infty}$. manifold is almost-parallel if the tensor $C$ of type $(2,2)$ over $R^{2}$ associated with 
it is a specialization of one or the other of two variable tensors $C^{* \prime} s$ whose components $\widetilde{C}^{*}$ arranged lexicographically as elements of a $2^{2} \times 2^{2}$ matrix are respectively as follows:

$$
\left(\begin{array}{cccc}
\cdot & \cdot & \cdot & \cdot \\
* & \cdot & \cdot & \cdot \\
* & \cdot & \cdot & \cdot \\
\cdot & * & * & \cdot
\end{array}\right),\left(\begin{array}{cccc}
\cdot & \cdot & \cdot & \cdot \\
\cdot & \cdot & \cdot & \cdot \\
\cdot & \cdot & \cdot & \cdot \\
* & \cdot & \cdot & \cdot
\end{array}\right),
$$

where a dot denotes the zero, and a star denotes an independent variable.

2. 6. Some other conditions for a recurrent teosor of type $(r, r)$ to be almostparallel

We recall from algebra that a square matrix $N$ is said to be nilpotent if $N^{m}=0$ for some integer $m>1$, and the smallest such integer $m$ is called the index of nilpotence. We shall introduce the concepts of nilpotence and complete nilpotence for tensors of type $(r, r)$.

Consider first a tensor $C$ of type $(r, r)$ over $R^{n}$. Let $C_{B}^{A}$ be the components of $C$ relative to some basis $\left\{e_{\alpha}\right\}$ of $R^{n}$. We say that $C$ is nilpotent of index $m$ if the matrix $\left(C_{B}^{A}\right)$ is nilpotent of index $m$. To justify this definition we must show that it is independent of the basis $\left\{e_{\alpha}\right\}$ of $R^{n}$ used. In fact, if $\left\{e_{\alpha^{\prime}}\right\}=$ $\left\{\boldsymbol{e}_{\alpha} g_{\alpha^{\prime}}^{\alpha}\right\}$ is another basis of $R^{n}$, then the components $C_{B^{\prime}}^{A^{\prime}}$ of $C$ relative to the basis $\left\{e_{\alpha^{\prime}}\right\}$ are

$$
C_{B^{\prime}}^{A^{\prime}}=\bar{g}_{A}^{A^{\prime}} C_{B}^{A} g_{B^{\prime}}^{B}
$$

Since $\left(\bar{g}_{A}^{A^{\prime}}\right)=\left(g_{B^{\prime}}^{B}\right)^{-1}$, the matrices $\left(C_{B^{\prime}}^{A^{\prime}}\right)$ and $\left(C_{B}^{A}\right)$ are similar, and so, they are either both non-nilpotent or both nilpotent with the same index.

We now write equation (2.6) as

$$
k_{A}^{C} C_{B}^{A} \bar{k}_{E}^{B}=\sigma C_{E}^{C},
$$

where $\sigma>0,\left(k_{A}^{C}\right)=\left(k_{\alpha_{1}}^{\Upsilon_{1}} \cdots k_{\alpha r}^{\Upsilon_{r}}\right)$ is a non-singular matrix of order $n^{r}$ and $\left(\bar{k}_{E}^{B}\right)$ $=\left(k_{A}^{c}\right)^{-1}$. Equating the characteristic determinants of the two sides of (2.15), we get

$$
\operatorname{det}\left(\lambda \delta_{B}^{A}-C_{B}^{A}\right)=\operatorname{det}\left(\lambda \delta_{B}^{A}-\sigma C_{B}^{A}\right) .
$$

When this is expressed in the polynomial form

$$
\lambda^{p}+a_{1} \lambda^{p-1}+\cdots+a_{p}=\lambda^{p}+a_{1} \sigma \lambda^{p-1}+\cdots+a_{p} \sigma^{p},
$$

where $p=n^{r}$, we see that 


$$
a_{1}=a_{1} \sigma, \cdots, \quad a_{p}=a_{p} \sigma^{p} .
$$

Since $\sigma>0$, it follows from these that either $\sigma=1$ or all the $a_{1}, \cdots, a_{p}$ are zero. But $a_{1}, \cdots, a_{p}$ are all zero iff the matrix $\left(C_{B}^{A}\right)$ is nilpotent. Hence we have proved

LEMMA 2.4. If a tensor $C$ of type $(r, r)$ over $R^{n}$ is consistent with some $\sigma \neq 1$, then it is nilpotent. In other words, if $C$ is not nilpotent, then it is consistent with only $\sigma=1$.

Let $D$ be any contraction of $C$, i.e. any tensor obtained from $C$ by contraction. Then $D$ is a tensor of type $(s, s)$. Let $D_{B_{1}}^{A_{1}}$ be the components of $D$, where we denote by $A_{i}, \cdots$ the composite indices $A_{1}=\left(\alpha_{1}, \cdots, \alpha_{s}\right), \cdots$ Then equation (2.15) implies that

$$
\begin{gathered}
k_{A_{1}}^{C_{1}} D_{B_{1}}^{A_{1}} \bar{k}_{E_{1}}^{B_{1}}=\sigma D_{E^{1}}^{r_{1}}, \\
k_{A}^{C} k_{A_{1}}^{C_{1}}\left(C_{B}^{A} D_{B_{1}}^{A_{1}}\right) \bar{k}_{E}^{B} \bar{k}_{E_{1}}^{R_{1}}=\sigma^{2} C_{E}^{C} D_{E_{1}}^{C_{1}} .
\end{gathered}
$$

It follows from these and Lemma 2.4 that if $C$ is consistent with some $\sigma>0$ and $\neq 1$, then $C$, its contractions, and the contractions of the (finite) tensor products of $C$ and its contractions are all nilpotent. This gives rise to the following definition and proves Proposition 2.3 below.

A tensor $C$ of type $(r, r)$ over $R^{n}$ is said to be completely nilpotent if the following tensors constructed from $C$ are all nilpotent:

(i) $C$ and its contractions.

(ii) The contractions of the (finite) tensor products of the tensors in (i) ${ }^{2}$.

Proposition 2.3. If a tensor $C$ of type $(r, r)$ over $R^{n}$ is consistent with some $\sigma>0$ and $\neq 1$, then $C$ is completely nilpotent. In other words, if $C$ is not completely nilpotent, then $C$ is consistent with only $\sigma=1$.

For tensors of type $(1,1)$, nilpotence implies complete nilpotence. In this case, we can prove

Proposition 2.4. A tensor $C$ of type $(1,1)$ over $R^{n}$ is either consistent with arbitary $\sigma>0$ or consistent with only $\sigma=1$. Furthermore, $C$ is consistent with arbitary $\sigma>0$ iff it is nilpotent.

Proof. For a tensor $C$ of type $(1,1)$, equation (2.6) becomes

2) In this respect we note that the tensor product of a tensor of type $(r, r)$ with a nilpotent tensor of type $(s, s)$ is nilpotent (a consequence of Lemma 3.1 (ii)). 


$$
k_{\alpha}^{\Upsilon} C_{\beta}^{\alpha} \bar{k}_{\varepsilon}^{\beta}=\sigma C_{\varepsilon}^{\Upsilon} \quad(1 \leq \alpha, \beta, \cdots \leq n) .
$$

As in the proof of Lemma 2.4, equation (2.16) implies that either the matrix $\left(C_{\beta}^{\alpha}\right)$ is nilpotent or $\sigma=1$. Thus, to prove Proposition 2.4, it suffices to show that if $\left(C_{\beta}^{\alpha}\right)$ is nilpotent, then the tensor $C$ is consistent with arbitrary $\sigma>0$, i.e., for any $\sigma>0$, there exists a $k \in G L(n, R)$ such that equation (2.16) is satisfied.

Let $\lambda_{1}, \ldots ;\left(\lambda-\lambda_{1}\right)^{e_{1}}, \cdots$ be respectively the characteristic roots and the elementary divisors of $\left(C_{\beta}^{\alpha}\right)$, and let $\sigma>0$ be any number. Then $\left(\lambda-\sigma \lambda_{1}\right)^{e_{1}}, \ldots$ are the elementary divisors of $\left(\sigma_{\sigma} C_{\beta}^{\alpha}\right)$. Since $\left(C_{\beta}^{\alpha}\right)$ is nilpotent, its characteristic roots are all zero. Therefore, the two matrices $\left(C_{\beta}^{\alpha}\right)$ and $\left(\sigma C_{\beta}^{\alpha}\right)$ have the same elementary divisors $\lambda^{e_{1}}, \ldots$ and consequently are similar. Hence, there exists some non-singular matrix $\left(k_{\alpha}^{\Upsilon}\right)$ with real elements such that $(2.16)$ is satisfied. This completes the proof of the proposition.

We now consider tensors on $M$. Let $S$ be a tensor of type $(r, r)$ on $M$, and $\boldsymbol{u}$ any point in $M$. Then $S(\boldsymbol{u})$ is a tensor of type $(\boldsymbol{r}, \boldsymbol{r})$ over the $\boldsymbol{n}$-dimensional tangent space to $M$ at $u$, and the definitions of nilpotence and complete nilpotence of tensors of type $(r, r)$ over $R^{n}$ apply to $S(u)$. We now show that if a parallel or recurrent tensor $S$ of type $(r, r)$ on $M$ is nilpotent of index $m$ (resp. completely nilpotent) at some point $u$ in $M$, then it is nilpotent of index $m$ (resp. completely nilpotent) everywhere in $M$. In fact, since $S$ is parallel or recurrent, there exists at each point $u \in M$ a frame $z(u)$ in $M$ such that

$$
S_{B}^{A}(z(u))=C_{B}^{A} \quad \text { or } \quad S_{B}^{A}(z(u))=\phi(z(u)) C_{B}^{\hat{A}} \equiv \rho(u) C_{B}^{A},
$$

where the $C_{B}^{A}$ are constants but not all zero, and $\rho(u)$ is nowhere zero in $M$. If $S$ is nilpotent of index $m$ (resp. completely nilpotent) at some point in $M$, then it follows from (2.17) that the matrix $\left(C_{B}^{A}\right)$ is nilpotent of index $m$ (resp. completely nilpotent). Hence by (2.17) again, $S$ is nilpotent of index $m$ (resp. completely nilpotent) everywhere in $M$.

A consequence of Proposition 2.3 and Theorem 2.3 is the following

THEOREM 2.7. A necessary condition for a recurrent tensor of type $(r, r)$ on $M$ to be almost-parallel is that it is completely nilpotent.

Proof. Let $S$ be a recurrent tensor of type $(r, r)$ on $M$ and $C$ the tensor over $R^{n}$ associated with it. Assume that $S$ is not completely nilpotent. Then by Proposition 2.3, $C$ is consistent with only $\sigma=1$. Therefore, by Theorem 2.3, 
$S$ is not almost-parallel, as was to be proved.

The following theorem is an immediate consequence of Proposition 2.4 and Theorem 2.3:

THEOREM 2.8. A recurrent tensor of type $(1,1)$ on $M$ is almost-parallel iff it is nilpotent.

Proposition 2.3 says that if $C$ is not completely nilpotent, then it is consistent with only $\sigma=1$. Using this fact and the method of proving Theorem 2.4, we can prove

THEOREM 2.9. For a recurrent tensor of type $(r, r)$ on $M$ which is not completely nilpotent, the recurrence covector is globally a gradient.

A direct and simpler proof of this theorem is the following. Since $S$ is recurrent, we have by definition

$$
\nabla S=W \otimes S, \quad W \neq 0 .
$$

Let $T$ be any one of the tensors constructed from $S$ in the manner described in the definition of complete nilpotence of $C$. Then $T$ satisfies the equation $\nabla T=W \otimes T$, and consequently also the equation

$$
\nabla T^{(q)}=q W \otimes T^{(q)},
$$

where $q$ is any positive integer and $T^{(q)}$ denotes the " $q$ th power" of $T$.

Since $S$ is not completely nilpotent, there exists at least one such tensor $T$ which is not nilpotent. For this $T$, there exists some positive integer $q$ and some point $u$ in $M$ such that the trace of $T^{(q)}(u)$ is not zero. On the other hand, it follows from (2.18) that

$$
\nabla\left(\operatorname{trace} T^{(q)}\right)=q\left(\operatorname{trace} T^{(q)}\right) W .
$$

Hence the $C^{\infty}$-function (trace $T^{(q)}$ ) on $M$ is nowhere zero, and $W$ is globally a gradient, as was to be proved.

It is an open question whether complete nilpotence is a sufficient condition for a recurrent tensor of type $(r, r), r \geq 2$, on $M$ to be almost-parallel (i.e. for a tensor $C$ of type $(r, r), r \geq 2$, over $R^{n}$ to be consistent with arbitrary $\left.\sigma>0\right)$. And algebraically, it would be an interesting problem to determine for each $r \geq 2$ all the completely nilpotent tensors of type $(r, r)$.

Other algebraic considerations of the consistency of equation (2.6) lead to 
the following theorem the proof of which will be given later in $\S 4$.

THEOREM 2.10. For a recurrent tensor of type $(r, r)$ to be almost-parallel it is necessary

(i) that it is nilpotent and its index of nilpotence is at most equal to

$$
(n+r-1) ! /(n-1) ! r ! ;
$$

(ii) that it has at least

$$
r ! /(q !)^{n}(q+1)^{p} \quad(r=n q+p, 0<p<q)
$$

elementary divisors.

Here the elementary divisors of a tensor $S$ of type $(r, r)$ at $u \in M$ is defined as the elementary divisors of the matrix $\left(S_{B}^{A}(z(u))\right)$. By arguments similar to those used in the first part of $\$ 2.6$, it can easily be shown that this definition is legitimate and that the number of elementary divisors of a parallel or recurrent tensor of type $(r, r)$ on $M$ is everywhere the same in $M$.

\section{7. Examples}

Some easy examples can be given to illustrate the theorems in this section. First let $M$ be any connected $C^{\infty}$-manifold, $g_{i j}$ a Riemannian metric on $M$ and $\rho$ a non-constant nowhere-zero $C^{\infty}$-function on $M$. Then the tensor $S_{i j}=\rho g_{i j}$ of type $(0,2)$ is recurrent with respect to the Riemannian connection arising from $g_{i j}$, while it is parallel with respect to the Riemannian connection arising from the metric tensor $S_{i j}$. On the other hand. the tensor $S_{j}^{i}=\rho \delta_{j}^{i}$ of type $(1,1)$ on $M$, where $\rho$ is as above and $\delta_{j}^{i}$ is the Kronecker delta, is recurrent with respect to every linear connection on $M$, but is never parallel with respect to any linear connection on $M$. Furthermore, for this recurrent tensor $S_{j}^{i}$, which is not nilpotent, the recurrence covector is $\partial_{l} \log \rho$ and is therefore globally a gradient.

Next, let $M$ be a connected parallelisable $C^{\infty}$-manifold, and $\left\{X_{\alpha}\right\}$ a $C^{\infty}$-field of frames on $M$. Then it is well known (cf. Eisenhart [3], p. 48) that

$$
\Gamma_{j k}^{i}=-x_{j}^{\alpha} \partial_{k} x_{\alpha}^{i}
$$

are local components of a linear connection $\Gamma$ on $M$ with respect to which each vector $X_{\alpha}$ is parallel. Now let $C_{B}^{A}$ be any set of $n^{2 r}$ constants not all zero, and $\rho$ any non-constant nowhere-zero $C^{\infty}$-function on $M$. Then the tensor $S$ of type $(r, r)$ on $M$ defined by the components 


$$
S_{J}^{I}=\rho x_{A}^{I} C_{B}^{A} x_{J}^{B}
$$

is such that $\nabla_{l} S_{J}^{\prime}=\left(\partial_{l} \log \rho\right) S_{J}^{I} . \quad S$ is therefore recurrent with respect to $\Gamma$. It is almost-parallel if, for example, the tensor $C$ over $R^{n}$ with components $C_{B}^{A}$ is a specialization of one of the variable tensors $C^{*}$ s determined in Proposition 2.2 ; it is not almost-parallel if, for example, the tensor $C$ is not completely nilpotent.

\section{The matrix equation $\left(\otimes^{r} k\right) C\left(\otimes^{r} k^{-1}\right)=\sigma C$, and proof of Proposition 2.2}

The object of this section is to prove Proposition 2.2 on the consistency of a tensor $C$ of type $(r, r)$ over $R^{n}$ with a real number $\sigma>0$ corresponding to which there is a $k \in G L(n, R)$ whose characteristic roots are all real. Let $\widetilde{C}=$ $\left(C_{\beta_{1} \ldots \beta_{r}}^{\alpha_{1} \ldots \alpha_{r}}\right.$ ) be the set of components of $C$ relative to some basis $\left\{e_{\alpha}\right\}$ in $R^{n}$. Then by definition, $C$ is consistent with $\sigma$ if the equation $k \cdot \widetilde{C}=\sigma \widetilde{C}$, i.e.

$$
k_{\alpha_{1}}^{\gamma_{1}} \cdots k_{\alpha_{r}}^{\gamma_{r}} C_{\beta_{1} \cdots \beta r}^{\alpha_{1} \cdots \alpha_{r}} \bar{k}_{\varepsilon_{1}}^{\beta_{1}} \cdots \bar{k}_{\varepsilon r}^{\beta r}=\sigma C_{\varepsilon_{1} \cdots \varepsilon_{r}}^{\gamma_{1} \ldots \gamma_{r}}
$$

holds for some non-singular matrix $\left(k_{a}^{\Upsilon}\right)$ with real elements.

Let $g$ be any element of $G L(n, R)$. Then, changing the basis $\left\{e_{\alpha}\right\}$ in $R^{n}$ to $\left\{e_{\alpha} \cdot g\right\}$ changes the equation $k \cdot \widetilde{C}=\sigma \widetilde{C}$ to $\left(g^{-1} k g\right) \cdot \widetilde{C}^{\prime}=\widetilde{C}^{\prime}$, where $\widetilde{C}^{\prime}$ is the set of components of $C$ relative to the basis $\left\{e_{\alpha} \cdot g\right\}$ (see $\S 2.3$ ). This change does not change the characteristic roots of $k$. Since we now confine ourselves to the case where the characteristic roots of $k$ are all real, we can always choose $g \in G L(n, R)$ so that the matrix of $g^{-1} k g$ is in Jordan canonical form. Therefore, to study the consistency of a tensor $C$ with a number $\sigma>0$ for which there exists a corresponding $k \in G L(n, R)$ whose characteristic roots are all real, it suffices to study the consistency of equation (3.1) in the case when the matrix $\left(k_{a}^{\Upsilon}\right)$ is in Jordan canonical form.

We shall treat equation (3.1) as a matrix equation by using the concept of direct products (i.e. Kronecker products) of matrices. It will be seen ( $\$ 3.4$ ) that the discussions in this section completely solve the problem of consistency of the equation $k \cdot \widetilde{C}=\sigma \widetilde{C}$ in an algebraically closed field.

3. 1. Direct sums and direct prodncts of matrices (cf. MacDuffee [8], pp. 8186).

We need a few definitions and results on matrices. 
If $L_{1}, L_{2}$ are two matrices of order $m_{1}, m_{2}$, the matrix

$$
L_{1} \oplus L_{2} \equiv\left[\begin{array}{cc}
L_{1} & 0 \\
0 & L_{2}
\end{array}\right]
$$

of order $m_{1}+m_{2}$ is called the direct sum of $L_{1}$ and $L_{2}$. If $L_{1}=\left(l_{b}^{a}\right),\left(1 \leq a, b \leq m_{1}\right)$, then the matrix

$$
L_{1} \otimes L_{2} \equiv\left(\begin{array}{cccc}
l_{1}^{1} L_{2} & \cdots & \cdot & l_{n_{1}}^{1} L_{2} \\
\cdot & \cdot & \cdot \\
\cdot & \cdot & \cdot \\
l_{1}^{n_{1}} L_{2} & \cdots & \cdot & l_{n_{1}}^{n_{1}} L_{2}
\end{array}\right)
$$

of order $m_{1} m_{2}$ is called the direct product of $L_{1}$ and $L_{2}$. Neither the direct sum nor the direct product is commutative. Direct sums and direct products of more than two matrices are defined in a natural way, and it is easily seen that they are both associative. In particular, we have the direct $r$ th power of a square matrix $L$, defined by

$$
\otimes^{r} L \equiv L \otimes \cdots \otimes L \quad(r \text { factors }) .
$$

In what follows, $s$ and $r$ are some fixed positive integers; the indices $\mu$, $\mu_{1}, \cdots, \mu_{r}$ all have the range $1, \cdots, s$; and matrices except the non-singular $\boldsymbol{n} \times \boldsymbol{n}$ matrix $k$ are denoted by capital latin letters. By a permutation matrix we mean a square matrix whose elements are one or zero and which has exactly one nonzero element in each row and exactly one in each column. We write $F \sim \widetilde{F}$ if the matrices $F$ and $\widetilde{F}$ are similar.

The following lemma contains some properties of the direct products of matrices. The proof will be omitted if it is obvious.

Lemma 3.1.

(i) $\left(L_{1}+L_{2}\right) \otimes L_{3}=L_{1} \otimes L_{3}+L_{2} \otimes L_{3}$ $L_{1} \otimes\left(L_{2}+L_{3}\right)=L_{1} \otimes L_{2}+L_{\mathrm{i}} \otimes L_{3}$.

(ii) $\left(L_{1} L_{2}\right) \otimes\left(L_{3} L_{4}\right)=\left(L_{1} \otimes L_{3}\right)\left(L_{2} \otimes L_{4}\right)$.

(iii) $\left(\oplus_{\mu} K_{\mu}\right) \otimes L=\oplus_{\mu}\left(K_{\mu} \otimes L\right)$.

(iv) $L \otimes\left(\oplus_{\mu} K_{\mu}\right) \sim \oplus_{\mu}\left(L \otimes K_{\mu}\right)$,

where the similarity can be accomplished by a permutation matrix which depends only on the orders of the matrices $L$ and $K_{\mu}$. 
(v) $K_{\mu} \sim \widetilde{K}_{\mu}$ imply $\oplus_{\mu} K_{\mu} \sim \oplus_{\mu} \widetilde{K}_{\mu}$ and $\otimes_{\mu} K_{\mu} \sim \otimes_{\mu} \widetilde{K}_{\mu}$.

(vi) $\left(\oplus_{\mu_{1}} K_{\mu_{1}}\right) \otimes \cdots \otimes\left(\oplus_{\mu_{r}} K_{\mu_{r}}\right) \sim \oplus_{\mu_{1}} \cdots \mu_{\mu_{r}}\left(K_{\mu_{1}} \otimes \cdots \otimes K_{\mu_{r}}\right)$,

where the similarity can be accomplished by a permutation matrix which depends only on the orders $n_{1}, \ldots, n_{s}$ of the matrices $K_{1}, \ldots, K_{s}$.

Proof of (iv). Let $L=\left(l_{d}^{c}\right),(1 \leq c, d \leq m)$. Then

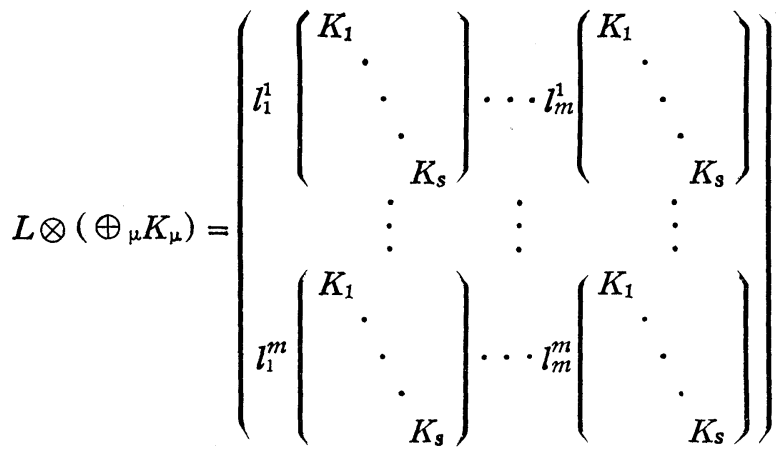

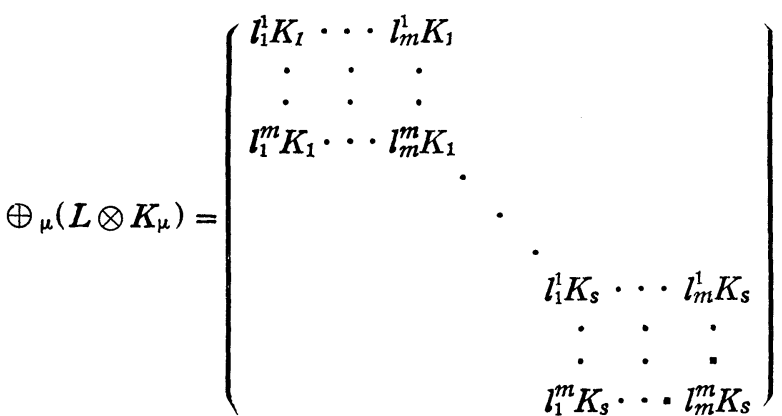

The two matrices on the right sides can be transformed one into the other by suitable rearrangements of the rows and the corresponding columns; they are therefore similar to each other under a permutation matrix which is easily seen to depend only on the orders of the matrices $L$ and $K_{\mu}$.

Proof of (vi). The formula is trivially true for $r=1$. By (iii), (iv) and (v), we have successively

$$
\begin{aligned}
\left(\oplus_{\mu_{1}} K_{\mu_{1}}\right) \otimes\left(\oplus_{\mu_{2}} K_{\mu_{2}}\right) & =\oplus_{\mu_{1}}\left\{K_{\mu_{1}} \otimes\left(\oplus_{\mu_{2}} K_{\mu_{2}}\right)\right\} \\
& =\oplus_{\mu_{1}}\left\{\sim \oplus_{\mu_{2}}\left(K_{\mu_{1}} \otimes K_{\mu_{2}}\right)\right\} \\
& \sim \oplus_{\mu_{1} \mu_{2}}\left(K_{\mu_{1}} \otimes K_{\mu_{2}}\right) .
\end{aligned}
$$

Therefore formula ( $v i$ ) is true for $r=2$. Now assume that it is true for $r \leq$ $m-1$. Then 


$$
\begin{aligned}
\left(\oplus_{\mu_{1}} K_{\mu_{1}}\right) \otimes \cdots \otimes\left(\oplus_{\mu_{m}} K_{\mu_{m}}\right)= & \left\{\left(\oplus_{\mu_{1}} K_{\mu_{1}}\right) \otimes \cdots \otimes\left(\oplus_{\mu_{m-1}} K_{\mu_{m-1}}\right)\right\} \otimes\left(\oplus_{\mu_{m}} K_{\mu_{m}}\right) \\
\text { (by induction hypothesis })= & \left.\sim \oplus_{\mu_{1}} \cdots \mu_{m-1}\left(K_{\mu_{1}} \otimes \cdots \otimes K_{\mu_{m-1}}\right)\right\} \otimes\left(\oplus_{\mu_{m}} K_{\mu_{m}}\right) \\
\text { (by Lemma 3.1 (v)) } & \sim\left\{\oplus_{\mu_{1}} \cdots \mu_{m-1}\left(K_{\mu_{1}} \otimes \cdots K_{\mu_{m-1}}\right)\right\} \otimes\left(\oplus_{\mu_{m}} K_{\mu_{m}}\right) \\
\text { (by formula (vi) for } r=2) & \sim \oplus_{\mu_{1}} \cdots \mu_{m}\left(K_{\mu_{1}} \otimes \cdots \otimes K_{\mu_{m-1}}\right) \otimes K_{\mu_{m}} \\
& =\oplus_{\mu_{1}} \cdots \mu_{m}\left(K_{\mu_{1}} \otimes \cdots \otimes K_{\mu_{m}}\right) .
\end{aligned}
$$

This completes the proof of formula (vi). The assertion that the similarity can be accomplished by a permutation matrix follows from the fact that direct sums and direct products of permutation matrices are permutation matrices.

We now prove

Lemma 3.2. Let $\lambda_{\mu}$ be any fixed numbers, $E_{\mu}$ the unit matrix of order $n_{\mu}$, and $N_{\mu}$ any nilpotent matrix of order $n_{\mu}$. Then the matrix

$$
N_{\mu_{1}} \cdots \mu_{k} \equiv\left(\lambda_{\mu_{1}} E_{\mu_{1}}+N_{\mu_{1}}\right) \otimes \cdots \otimes\left(\lambda_{\mu_{r}} E_{\mu_{r}}+N_{\mu_{r}}\right)-\lambda_{\mu_{1}} \cdots \lambda_{\mu_{r}} E_{\mu_{1}} \otimes \cdots \otimes E_{\mu_{r}}
$$

of order $n_{\mu_{1}} \cdots n_{\mu_{r}}$ is nilpotent.

Proof. If we expand the first term on the right side of the above equation by using Lemma 3.1 (i), we see that $N_{\mu 1} \ldots \mu_{r}$ is the sum of a finite number of matrices each of which is a direct product containing at least one of the nilpotent matrices $N_{\mu}$ as factor. But an easy consequence of Lemma 3.1 (ii) is that a direct product containing a nilpotent matrix as factor is itself nilpotent. Therefore, $N_{\mu_{1}} \ldots \mu_{r}$ is the sum of nilpotent matrices and is consequently a nilpotent matrix itself.

\section{2. Proof of Proposition 2. 2}

We now confine ourselves to the field of real numbers. Let us first fix the notation and give a few definitions. Denote by $k$ the square non-singular matrix of order $n$ with elements $k_{\beta}^{\varepsilon}$, and by $C$ the squaer matrix of order $n^{r}$ with elements $C_{\beta_{1} \ldots \beta r}^{\alpha_{1} \ldots \alpha_{r}}$ arranged in the lexicographical order. Then equation (3.1) can be written as

$$
\left(\otimes^{r} k\right) C\left(\otimes^{r} k^{-1}\right)=\sigma C,
$$

where the left side is the (ordinary) product of the matrices $\otimes^{r} k, C, \otimes{ }^{r} k^{-1}$ of order $n^{r}$. Corresponding to the definitions given in $\$ 2.5$ for tensors of type $(r, r)$ over $R^{n}$, we shall say that a matrix $C$ of constants (i.e. a matrix $C$ whose elements are constants) is consistent with the number $\sigma>0$ if equation (3.2) holds for some non-singular matrix $k$. A matrix $C^{*}=\left(C_{\beta_{1} \ldots \beta_{r}}^{* \alpha_{1} \ldots \alpha_{r}}\right)$ is called a 
matrix of variables if its non-zero elements are independent variables. A matrix $C$ of constants is called a specialization of the matrix $C^{*}$ of variables if $C$ is obtained from $C^{*}$ by giving constant values to the variable elements contained in $C^{*}$. It is obvious that if for some $k$ and $\sigma$ equation (3.2) is satisfied by a variable matrix $C^{*}$, then it is satisfied by any specialization $C$ of $C^{*}$.

Three lemmas are needed for the proof of Proposition 2.2. In what follows, we assume that $s$ is some fixed integer such that $1 \leq s \leq n$; the indices $\mu, \mu_{1}$, $\cdots, \mu_{r}, \nu_{1}, \cdots, \nu_{r}$ all have the range $1, \cdots, s ; \lambda_{\mu}$ are some fixed (real) numbers; $E_{\mu}$ is the unit matrix of order $n_{\mu}$ such that $\Sigma_{\mu} n_{\mu}=n$; and $N_{\mu}$ is the nilpotent matrix of order $n_{\mu}$ and index $n_{\mu}$ in Jordan canonical form (i.e. all its elements on the first superdiagonal are 1 and all the other elements are 0 ).

\section{LEMMA 3.3. Let the equation}

$$
\left(\otimes^{r} k\right) C\left(\otimes^{r} k^{-1}\right)=\sigma C
$$

be satisfied by

$$
C=C_{0} \neq 0, \quad \sigma=\sigma_{0}>0, \quad k=\oplus_{\mu}\left(\lambda_{\mu} E_{\mu}+N_{\mu}\right) \quad\left(\lambda_{\mu} \neq 0\right)
$$

and let $C=C_{0}^{*}$ be the most general solution of (3.2) when

$$
\sigma=\sigma_{0}, \quad k=\oplus_{\mu}\left(\lambda_{\mu} E_{\mu}\right) .
$$

Then $C_{0}^{*}$ is a matrix of variables and $C_{0}$ is a specialization of $C_{0}^{*}$.

Proof. Let us study equation (3.2) when

$$
\sigma=\sigma_{0}, \quad k=\oplus_{\mu} K_{\mu}=\oplus_{\mu}\left(\lambda_{\mu} E_{\mu}+e_{\mu} N_{\mu}\right),
$$

where the $e_{\mu}$ are all equal to 1 or are all 0. By Lemma 3.1 (iv) we have

$$
\otimes^{r} k=L^{-1}\left(\oplus_{\mu_{1}} \ldots \mu_{r} K_{\mu_{1}} \otimes \cdots \otimes K_{\mu_{r}}\right) L,
$$

where $L$ is a permutation matrix of order $n^{r}$ which depends only on $n_{1}, \cdots, n_{s}$. Therefore equation (3.2) can be written

$$
\left(\oplus_{\mu_{1}} \cdots \mu_{r} K_{\mu_{1}} \otimes \cdots \otimes K_{\mu_{r}}\right) L C L^{-1}=\sigma_{0} L C L^{-1}\left(\oplus_{\mu_{1}} \cdots \mu_{r} K_{\mu_{1}} \otimes \cdots \otimes K_{\mu_{r}}\right) .
$$

Thus putting

$$
Z \equiv L C L^{-1}
$$

equation (3.2) is equivalent to

$$
\left(\oplus_{\mu_{1}} \cdots \mu_{r} K_{\mu_{1}} \otimes \cdots \otimes K_{\mu_{r}}\right) Z=\sigma_{0} Z\left(\oplus_{\mu_{1}} \cdots \mu_{r} K_{\mu_{1}} \otimes \cdots \otimes K_{\mu_{r}}\right) .
$$


We note that since $L$ is a permutation matrix, $C$ can be obtained from $Z$ by a rearrangement of its rows and the corresponding columns.

To study equation (3.3), we partition $Z$ into blocks

$$
Z=\left(Z_{\nu_{1} \cdots \nu_{r}}^{\mu_{1} \ldots \mu_{r}}\right), \quad\left(1 \leq \mu_{1}, \cdots, \mu_{r}, \nu_{1}, \cdots, \nu_{r} \leq s\right),
$$

corresponding to the partition $\left(\oplus_{\mu_{1}} \cdots \mu_{r} K_{\mu_{1}} \otimes \cdots \otimes K_{\mu_{r}}\right)$. Then $Z_{\nu_{1} \ldots \nu_{r}}^{\mu_{1} \cdots \mu_{r}}$ is an $\left(n_{\mu_{1}} \cdots n_{\mu_{r}}\right) \times\left(n_{\nu_{1}} \cdots n_{\nu_{r}}\right)$ matrix and (3.3) is equivalent to the following set of equations

$$
\left(K_{\mu_{1}} \otimes \cdots \otimes K_{\mu_{r}}\right) Z_{\nu_{1} \cdots \nu_{r}}^{\mu_{1} \cdots \mu_{r}}=\sigma_{0} Z_{\nu_{1} \cdots \nu_{r}}^{\mu_{1} \cdots \mu_{r}}\left(K_{\nu_{1}} \otimes \cdots \otimes K_{\nu_{r}}\right) .
$$

Here and in similar equations appearing below, no summation is assumed over the repeated indices.

Now by Lemma 3.1 (i),

$$
\begin{aligned}
K_{\mu_{1}} \otimes \cdots \otimes K_{\mu_{r}} & =\left(\lambda_{\mu_{1}} E_{\mu_{1}}+e_{\mu_{1}} N_{\mu_{1}}\right) \otimes \cdots \otimes\left(\lambda_{\mu_{r}} E_{\mu_{r}}+e_{\mu_{r}} N_{\mu_{r}}\right) \\
& =\lambda_{\mu_{1}} \cdots \lambda_{\mu_{r}} E_{\mu_{1}} \otimes \cdots \otimes E_{\mu_{r}}+N_{\mu_{1}} \cdots \mu_{r},
\end{aligned}
$$

where

$$
\begin{aligned}
N_{\mu_{1}} \cdots \mu_{r} & \equiv\left(\lambda_{\mu_{1}} E_{\mu_{1}}+\boldsymbol{e}_{\mu_{1}} N_{\mu_{1}}\right) \otimes \cdots \otimes\left(\lambda_{\mu_{r}} E_{\mu_{r}}+\boldsymbol{e}_{\mu_{r}} N_{\mu_{r}}\right) \\
& -\lambda_{\mu_{1}} \cdots \lambda_{\mu_{r}} E_{\mu_{1}} \otimes \cdots \otimes E_{\mu_{r}} .
\end{aligned}
$$

On account of this and the fact that $E_{\mu_{1}} \otimes \cdots \otimes E_{\mu_{r}}$ is equal to the unit matrix of order $n_{\mu_{1}} \cdots n_{\mu_{r}}$, equation (3.4) can be written

$$
\left(\sigma_{0} \lambda_{\nu_{1}} \cdots \lambda_{\nu_{r}}-\lambda_{\mu_{1}} \cdots \lambda_{\mu_{r}}\right) Z_{\nu_{1} \ldots \nu_{r}}^{\mu_{1} \cdots \mu_{r}}=N_{\mu_{1}} \ldots \mu_{r} Z_{\nu_{1} \ldots \nu_{r}}^{\mu_{1} \ldots \mu_{r}}-\sigma_{0} Z_{\nu_{1} \ldots \nu_{r}}^{\mu_{1} \ldots \mu_{\nu_{1}}} F_{\nu_{1}} \cdots \nu_{r}
$$

There are two cases to be considered.

Case 1. $\sigma_{0} \lambda_{\nu_{1}} \cdots \lambda_{\nu_{r}}-\lambda_{\mu_{1}} \cdots \lambda_{\mu_{r}} \neq 0$. Iterating equation $(3.5)(m-1)$-times, we get

$$
\begin{aligned}
\left(\sigma_{0} \lambda_{\nu_{1}} \cdots \lambda_{\nu_{r}}\right. & \left.-\lambda_{\mu_{1}} \cdots \lambda_{\mu_{r}}\right)^{m} Z_{\nu_{1} \ldots \nu_{r}}^{\mu_{1} \ldots \mu_{r}} \\
& =\Sigma(-1)^{m_{1}}\left(\begin{array}{l}
m \\
m_{1}
\end{array}\right)\left(\sigma_{0}\right)^{m_{1}}\left(N_{\mu_{1}} \cdots \mu_{r}\right)^{m_{2}} Z_{\nu_{1} \ldots \nu_{r}}^{\mu_{1} \ldots \mu_{r}}\left(N_{\nu_{1}} \cdots \nu_{r}\right)^{m_{1}},
\end{aligned}
$$

where the summation is taken over the integers $m_{1}, m_{2}$ such that $m_{1}+m_{2}=m$ and $0 \leq m_{1}, m_{2} \leq m$, and $\left(\begin{array}{c}m \\ m_{1}\end{array}\right)$ are binomial coefficients. Since by Lemma 3.2 , $N_{\mu_{1}} \ldots \mu_{r}$ and $N_{\nu_{1}} \ldots \nu_{r}$ are nilpotent, the right side of the above equation is zero for sufficiently large $m$. Therefore, in this case, $Z_{\nu_{1} \ldots \nu_{r}}^{\mu_{1} \ldots \mu_{r}}=0$. 
Case 2. $\sigma_{0} \lambda_{\nu_{1}} \cdots \lambda_{\nu_{r}}-\lambda_{\mu_{1}} \cdots \lambda_{\mu_{r}}=0$. In this case, equation (3.5) reduces to

$$
N_{\mu_{1}} \ldots \mu_{r} Z_{v_{1} \ldots \nu_{r}}^{\mu_{1} \ldots \mu_{r}}=\sigma_{0} Z_{\nu_{1} \ldots \nu_{r}}^{\mu_{1} \ldots \mu_{r}} N_{\nu_{1}} \ldots \nu_{r} .
$$

When all the $e_{\mu}$ are equal to 1 , these equations generally impose some con ditions on $Z_{\nu_{1} \ldots \nu_{r}}^{\mu_{1} \ldots \mu_{r}}$. But when all the $e_{\mu}$ are zero, the matrices $N_{\mu_{1}} \ldots \mu_{r}$ and $N_{\nu_{1}} \ldots \nu_{r}$ are both zero so that the above equations all reduce to $0=0$; therefore, in this case, $Z_{\nu_{1} \ldots \nu_{r}}^{\mu_{1} \ldots \mu_{r}}$ are arbitrary.

From the above properties of $Z_{\nu_{1} \ldots \nu_{r}}^{\mu_{1} \ldots \mu_{r}}$ it follows that $L C_{0}^{*} L^{-1}$ is a matrix of variables and that $L C_{0} L^{-1}$ is a specialization of $L C_{0}^{*} L^{-1}$. Since $L$ is a permutation matrix, what has just been said of the matrices $L C_{0}^{*} L^{-1}$ and $L C_{0} L^{-1}$ hold for the matrices $C_{0}^{*}$ and $C_{0}$. Lemma 3.3 is thus completely proved.

LEMMA 3.4. For any fixed number $\sigma>0$ and any fixed diagonal matrix $k=$ $\oplus_{\alpha} \lambda_{\alpha}\left(\lambda_{\alpha} \neq 0,1 \leq \alpha, \beta \leq n\right)$, the most general solution of (3.2) for $C$ is the matrix $C^{*}$ of variables whose element

$$
C_{\beta_{1} \ldots \beta r}^{* \alpha_{1} \ldots \alpha_{r}}=0 \text { or an independent variable }
$$

according as

$$
\sigma \lambda_{\beta_{1}} \cdots \lambda_{\beta_{r}}-\lambda_{\alpha_{1}} \cdots \lambda_{\alpha_{r}} \neq 0 \text { or }=0 .
$$

Proof. For $k=\oplus_{\alpha} \lambda_{\alpha}$, we have

$$
\otimes^{r} k=\oplus_{\alpha_{1}} \cdots \alpha_{r}\left(\lambda_{\alpha_{1}} \cdots \lambda_{\alpha_{r}}\right),
$$

and the equation

$$
\left(\otimes^{r} k\right) C=\sigma C\left(\otimes^{r} k\right)
$$

is equivalent to

$$
\left(\sigma \lambda_{\beta_{1}} \cdots \lambda_{\beta_{r}}-\lambda_{\alpha_{1}} \cdots \lambda_{\alpha_{r}}\right) C_{\beta_{1} \ldots \beta_{r}}^{\alpha_{1} \ldots \alpha_{r}}=0,
$$

where no summation is taken over the indices $\alpha_{1}, \ldots, \alpha_{r}$, Lemma 3.4 is an immediate consequence of this.

Lemma 3.5. Let $\sigma>0$ be a parameter. Then any set of $(n-1)$ independent and consistent equations of the form

$$
\sigma \lambda_{\beta_{1}} \cdots \lambda_{\beta_{r}}=\lambda_{\alpha_{1}} \cdots \lambda_{\alpha_{r}}
$$

together with the condition $\lambda_{1} \cdots \lambda_{n} \vDash 0$, completely determines the ratios of 
$\lambda_{1}, \cdots, \lambda_{n}$ in the form

$$
\lambda_{1}: \cdots: \lambda_{n}=\varepsilon_{1} \sigma^{q_{1}}: \cdots: \varepsilon_{n} \sigma^{q_{n}},
$$

where each $\varepsilon_{1}, \cdots, \varepsilon_{n}$ is +1 or -1 , and $q_{1}, \cdots, q_{n}$ are rational numbers.

Proof. Let there be given $n-1$ independent and consistent equations of the form (3.6). Each of these equations can be written in the form

$$
f_{2}^{a_{2}} \cdots f_{n}^{a_{n}}=\sigma
$$

where $f_{2}=\lambda_{2} / \lambda_{1}, \ldots, f_{n}=\lambda_{n} / \lambda_{1}$ and each $a_{2}, \ldots, a_{n}$ is $-1,0$, or +1 , and, because $\sigma$ is a parameter, not all $a_{2}, \ldots, a_{n}$ are zero. If we eliminate $f_{n}$ from the $n-1$ equations of the form (3.7) which are equivalent to the $n-1$ given equations. we obtain $n-2$ independent and consistent equations of the form

$$
f_{2}^{b_{2}} \cdots f_{n-1}^{b_{n-1}}=\sigma^{b_{0}}
$$

where each $b_{0}, b_{2}, \cdots, b_{n-1}$ is an integer or zero, but not all $b_{2}, \cdots, b_{n-1}$ are zero. Repeating this process of elimination $n-3$ times, we arrive in the end at a single equation of the form

$$
f_{2}^{m_{2}}=\sigma^{m_{0}},
$$

where $m_{2}, m_{0}$ are integers, and $m_{2}$ is not zero because $\sigma$ is a parameter. This proves our lemma.

We are now ready to prove Proposition 2.2. Let us return to Lemma 3.3 and write the matrix $\oplus_{\mu}\left(\lambda_{\mu} E_{\mu}\right)$ there as $\oplus_{\alpha} \lambda_{0 \alpha}=\operatorname{diag}\left(\lambda_{01}, \cdots, \lambda_{0 n}\right)$, where $1 \leq \alpha, \beta \leq n$. Since $C_{0} \neq 0$, so $C_{0}^{*} \neq 0$. Therefore, by Lemma 3.4, $\sigma_{0}$ and $\lambda_{0 \alpha}$ must satisfy one or more equations of the form

$$
\sigma \lambda_{\beta_{1}} \cdots \lambda_{\beta_{r}}=\lambda_{\alpha_{1}} \cdots \lambda_{\alpha_{r}} .
$$

Denote by $(*)_{0}$ the set of independent equations of this form satisfied by $\sigma_{0}$ and $\lambda_{0 \alpha}$. Since $\sigma_{0}>0$ and $\neq 1$, and $\lambda_{0 \alpha} \neq 0$, there exist, for each $\alpha$, a unique $\varepsilon_{0 \alpha}=+1$ or -1 , and a unique number $q_{0 \alpha}$ such that $\lambda_{0 \alpha}=\varepsilon_{0 \alpha} \sigma_{0}^{q_{0 \alpha}}$. Then the equations $(*)_{0}$, being satisfied by $\sigma=\sigma_{0}$ and $\lambda_{\alpha}=\varepsilon_{0 \alpha} \sigma_{0}^{q_{0 \alpha}}$, are satisfied by $\lambda_{\alpha}=\varepsilon_{0 \alpha} \sigma^{q_{0 x}}$ for arbitrary $\sigma>0$. From this it follows that the equation

$$
\left(\otimes^{r} k(\sigma)\right) C_{0}^{*}\left(\otimes^{r} k(\sigma)^{-1}\right)=\sigma C_{0}^{*}
$$

holds for

$$
k(\sigma)=\operatorname{diag}\left(\varepsilon_{01} \sigma^{q_{01}}, \cdots, \varepsilon_{0 n} \sigma^{q_{0 n}}\right) \text { and arbitrary } \sigma>0 .
$$


And since $C_{0}$ is a specialization of $C_{0}^{*}$, the same is true of the equation

$$
\left(\otimes{ }^{r} k(\sigma)\right) C_{0}\left(\otimes^{r} k(\sigma)^{-1}\right)=\sigma C_{0} .
$$

This proves the first part of Proposition 2.2.

To prove the second part of Proposition 2.2, we observe that the set $(*)_{0}$ of equations satisfied by $\sigma_{0}$ and $\lambda_{n \alpha}$ can always be extended in some way to a maximal set $(*)$ of $n-1$ independent and consistent equations of the form (3.6) with $\sigma>0$ as parameter. By Lemma 3.5, the most general solution of $(*)$ is of the form

$$
\lambda_{1}: \cdots: \lambda_{n}=\varepsilon_{1} \sigma^{q_{1}}: \cdots: \varepsilon_{n} \sigma^{q_{n}}
$$

where each $\varepsilon_{\alpha}$ is +1 or -1 and $q_{1}, \ldots, q_{n}$ are rational numbers. Now by Lemma 3.4 , for

$$
k(\sigma)=\operatorname{diag}\left(\varepsilon_{1} \sigma^{q_{1}}, \ldots, \varepsilon_{n} \sigma^{q_{n}}\right) \text { and arbitrary } \sigma>0,
$$

the most general solution for $C$ of the equation

$$
\left(\otimes^{r} k(\sigma)\right) C\left(\otimes^{r} k(\sigma)^{-1}\right)=\sigma C
$$

is a matrix $C^{*}$ of variables. And since the set $(*)_{0}$ of equations is contained in the set $(*)$ of equations, $C_{0}^{*}$ is a special case of $C^{*}$. But $C_{0}$ is a specialization of $C_{0}^{*}$. Therefore $C_{0}$ is a specialization of $C^{*}$. This completes the proof of Proposition 2.2.

\section{3. The complete set of $C^{*}$ 's for small $n$ and $r$}

As examples, we now determine the complete set of the variable tensors $C^{*}$ 's in Proposition 2.2 for a few simple cases. It should be noted that when two sets of $n-1$ independent and consistent equations of the form (3.6) determine two sets of ratios of $\lambda_{1}, \cdots, \lambda_{n}$ which differ from each other only by a permutation, they give rise to two matrices of variables whose elements are components, relative to two different bases, of the same variable tensor $C^{*}$ over $R^{n}$; for then the two corresponding matrices $k$ 's are similar under a permutation matrix (see the introductory remarks at the beginning of $\S 3$ ). It is not known whether, even after allowance has been made for the permutations of $\lambda_{1}, \cdots, \lambda_{n}$, the variable tensors determined in Proposition 2.2 are all distinct. But we shall not pursue this question as its answer is of no importance to our present problem. 
(i) The case $n=2, r=2$.

Here $1 \leq \alpha_{1}, \alpha_{2}, \beta_{1}, \beta_{2} \leq 2$ and the composite indices $A=\left(\alpha_{1}, \alpha_{2}\right), B=\left(\beta_{1}, \beta_{2}\right)$ have the range (11), (12), (21), (22). Among the equations of the form $\sigma \lambda_{\beta_{1}} \lambda_{\beta_{2}}$ $=\lambda_{\alpha_{1}} \lambda_{\alpha_{2}}$ we need only consider

$$
\sigma \lambda_{1}^{2}=\lambda_{1} \lambda_{2} ; \sigma \lambda_{1}^{2}=\lambda_{2}^{2},
$$

which give respectively,

$$
\lambda_{1}: \lambda_{2}=1: \sigma ; \lambda_{1}: \lambda_{2}=1: \sigma^{1 / 2} .
$$

Since the element $C_{\beta_{1} \beta_{2}}^{* \alpha_{1} \alpha_{2}}$ of $C^{*}$ is zero or an independent variable according as $\sigma \lambda_{\beta_{1}} \lambda_{\beta_{2}}-\lambda_{\alpha_{1}} \lambda_{\alpha_{2}} \neq 0$ or $=0$, we can easily see that the $C^{*}$ corresponding to these two equations are respectively

$$
\left(\begin{array}{cccc}
\cdot & \cdot & \cdot & \cdot \\
* & \cdot & \cdot & \cdot \\
* & \cdot & \cdot & \cdot \\
\cdot & * & * & .
\end{array}\right), \quad\left(\begin{array}{llll}
\cdot & \cdot & \cdot & \cdot \\
\cdot & \cdot & \cdot & \cdot \\
\cdot & \cdot & \cdot & \cdot \\
* & \cdot & \cdot & .
\end{array}\right),
$$

where a dot $\cdot$ denotes the zero and the stars $*$ denote independent variables.

(ii) The case $n=2, r=3$.

Here $1 \leq \alpha_{1}, \alpha_{2}, \alpha_{3}, \beta_{1}, \beta_{2}, \beta_{3} \leq 2$ and the composite indices $A=\left(\alpha_{1}, \alpha_{2}, \alpha_{3}\right)$, $B=\left(\beta_{1}, \beta_{2}, \beta_{3}\right)$ have the range (111), (112), (121), (122), (211), (212), (221), (222). Among the equations of the form $\sigma \lambda_{\beta_{1}} \lambda_{\beta_{2}} \lambda_{\beta_{3}}=\lambda_{\alpha_{1}} \lambda_{\alpha_{2}} \lambda_{\alpha_{3}}$, we need only consider

$$
\sigma \lambda_{1}^{3}=\lambda_{1}^{2} \lambda_{2} ; \sigma \lambda_{1}^{3}=\lambda_{1} \lambda_{2}^{2} ; \sigma \lambda_{1}^{3}=\lambda_{2}^{3},
$$

which give respectively

$$
\lambda_{1}: \lambda_{2}=1: \sigma ; \quad \lambda_{1}: \lambda_{2}=1: \sigma^{1 / 2} ; \quad \lambda_{1}: \lambda_{2}=1: \sigma^{1 / 3} .
$$

The corresponding $C^{*}$ are :

$$
\left(\begin{array}{cccccccc}
\cdot & \cdot & \cdot & \cdot & \cdot & \cdot & \cdot & \cdot \\
* & \cdot & \cdot & \cdot & \cdot & \cdot & \cdot & \cdot \\
* & \cdot & \cdot & \cdot & \cdot & \cdot & \cdot & \cdot \\
\cdot & * & * & \cdot & * & * & \cdot & \cdot \\
* & \cdot & \cdot & \cdot & \cdot & \cdot & \cdot & \cdot \\
\cdot & * & * & \cdot & * & * & \cdot & \cdot \\
\cdot & * & * & \cdot & * & * & \cdot & \cdot \\
\cdot & \cdot & \cdot & * & \cdot & \cdot & * & \cdot
\end{array}\right),\left(\begin{array}{cccccccc}
\cdot & \cdot & \cdot & \cdot & \cdot & \cdot & \cdot & \cdot \\
\cdot & \cdot & \cdot & \cdot & \cdot & \cdot & \cdot & \cdot \\
\cdot & \cdot & \cdot & \cdot & \cdot & \cdot & \cdot & \cdot \\
* & \cdot & \cdot & \cdot & \cdot & \cdot & \cdot & \cdot \\
\cdot & \cdot & \cdot & \cdot & \cdot & \cdot & \cdot & \cdot \\
* & \cdot & \cdot & \cdot & \cdot & \cdot & \cdot & \cdot \\
* & \cdot & \cdot & \cdot & \cdot & \cdot & \cdot & \cdot \\
\cdot & * & * & \cdot & * & \cdot & \cdot & \cdot
\end{array}\right)\left(\begin{array}{cccccccc}
\cdot & \cdot & \cdot & \cdot & \cdot & \cdot & \cdot & \cdot \\
\cdot & \cdot & \cdot & \cdot & \cdot & \cdot & \cdot & \cdot \\
\cdot & \cdot & \cdot & \cdot & \cdot & \cdot & \cdot & \cdot \\
\cdot & \cdot & \cdot & \cdot & \cdot & \cdot & \cdot & \cdot \\
\cdot & \cdot & \cdot & \cdot & \cdot & \cdot & \cdot & \cdot \\
\cdot & \cdot & \cdot & \cdot & \cdot & \cdot & \cdot & \cdot \\
\cdot & \cdot & \cdot & \cdot & \cdot & \cdot & \cdot & \cdot \\
* & \cdot & \cdot & \cdot & \cdot & \cdot & \cdot & \cdot
\end{array}\right)
$$


(iii) The case $n=3, r=2$.

Here $1 \leq \alpha_{1}, \alpha_{2}, \beta_{1}, \beta_{2} \leq 3$ and the composite indices $A=\left(\alpha_{1}, \alpha_{2}\right), B=\left(\beta_{1}, \beta_{2}\right)$ have the range (11), (12), (13), (21), (22), (23), (31), (32), (33). Each $C^{*}=$ $\left(C_{\beta_{1} \beta_{2}}^{* \alpha_{1} \alpha_{2}}\right)$ is a $9 \times 9$ matrix. There are altogether $15 C^{* \prime}$ s. Instead of listing them here, we only point out that each $C^{*}$ is determined from a set of two independent and consistent equations of the form $\sigma \lambda_{\beta_{1}} \lambda_{\beta_{2}}=\lambda_{\alpha_{1}} \lambda_{\alpha_{2}}$ such as

$$
\sigma \lambda_{1}^{2}=\lambda_{2} \lambda_{3}, \quad \sigma \lambda_{1} \sigma_{2}=\lambda_{3}^{2},
$$

which give

$$
\lambda_{1}: \lambda_{2}: \lambda_{3}=1: \sigma^{1 / 3}: \sigma^{2 / 3} \text {. }
$$

\section{4. The equation $k \cdot \widetilde{C}=\sigma \widetilde{C}$ in an algebraically closed field}

It is easily seen that the discussions of the equation $k \cdot \ddot{C}=\sigma \widetilde{C}$ in this section completely solve the problem of consistency of this equation in any algebraically closed field. In the case of field of real numbers, we have to confine ourselves to the case where the equation $k \cdot \widetilde{C}=\sigma \widetilde{C}$ is satisfied by some $\sigma>0$ and $\neq 1$ and some $k \in G L(n, R)$ whose characteristic roots are all real. The reason for this restriction is to ensure the existence of an element $g \in$ $G L(n, R)$ so that the matrix $g^{-1} k g$ is in Jordan canonical form. In the case of an algebraically closed field $F$, there is no need for any such restriction. Since all the characteristic roots of any $k \in G L(n, F)$ belong to $F$, there always exists some $\mathrm{g} \in G L(n, F)$ so that $g^{-1} \mathrm{~kg}$ is in Jordan canonical form. (Here we denote by $G L(n, F)$ the $n$-dimensional general linear group over the algebraically closed field $F$ ). Moreover, the content of $\S \S 3.1-3.3$ obviously holds for any field. Thus, we have as a by-product the following remarkable and perhaps important results :

Proposition 3.1. Let $F$ be any algebraically closed field. Let $C$ be a tensor of type $(r, r)$ over the $n$-dimensional vector space over $F$, and o a non-zero element of $F . C$ is said to be consistent with $\sigma$ if there exists an element $k$ of $G L(n, F)$ such that $k \cdot \widetilde{C}=\sigma \widetilde{C}$, where $\widetilde{C}$ is a set of components of $C$.

Then any given $C$ is consistent either with arbitrary $\sigma \neq 0$ or with only $\sigma=1$. Furthermore, $C$ is consistent with arbitrary $\sigma \neq 0$ iff it is a specialization of one of the variable tensors $C^{*}$ 's determined as follows:

(i) Let $\sigma \neq 0$ be a jarameter. Take any set (*) of $n-1$ independent and con. sistent equations of the form 


$$
\sigma \lambda_{\beta_{1}} \cdots \lambda_{\beta_{r}}=\lambda_{\alpha_{1}} \cdots \lambda_{\alpha_{r}}
$$

in the $n$ unknowns $\lambda_{1}, \cdots, \lambda_{n}$.

(ii) Solve $(*)$ for the ratios of $\lambda_{1}, \ldots, \lambda_{n}$, obtaining

$$
\lambda_{1}: \cdots: \lambda_{n}=\sigma^{q_{1}}: \cdots: \sigma^{q_{n}},
$$

where $q_{1}, \ldots, q_{n}$ are rational numbers.

(iii) Then the variable tensor $C^{*}$ corresponding to (*) is obtained by putting

$$
C_{\varepsilon_{1} \ldots \varepsilon_{r}}^{r_{1} \ldots \Upsilon_{r}}=0 \text { or an independent variable }
$$

according as the $\lambda_{1}, \cdots, \lambda_{n}$ given in (ii) satisfy

$$
\sigma \lambda_{\varepsilon_{1}} \cdots \lambda_{\varepsilon_{r}}-\lambda_{r_{1}} \cdots \lambda_{r_{r}} \neq 0 \text { or }=0
$$

\section{Proof of Theorem 2. 10}

The proof is based on certain algebraic considerations of the equation (3.2).

\subsection{Two lemmas}

Consider the equation (3.2) namely

$$
\left(\otimes^{r} k(\sigma)\right) C\left(\otimes^{r} k(\sigma)^{-1}\right)=\sigma C,
$$

where $C$ is a $n^{r} \times n^{r}$ matrix whose elements are constants, $\sigma>0$ is a parameter, and $k(\sigma)$ is a non-singular matrix of order $n$. Let $N$ be the Jordan canonical form of $C$. Then there exists a non-singular constant matrix $T$ such that

$$
T C T^{-1}=N \text {. }
$$

We note that since $C$ is nilpotent (see $\S 2.6$ ), $N$ has real elements, so that $T$ can be chosen to have real elements. Now using (4.2), we can write (4.1) as

$$
\left\{T^{-1}\left(\otimes^{r} k(\sigma)\right) T\right\} N\left\{T^{-1}\left(\otimes^{r} k(\sigma)^{-1}\right) T\right\}=\sigma N .
$$

Therefore, the matrix

$$
F(\sigma)=T^{-1}\left(\otimes^{r} k(\sigma)^{-1}\right) T
$$

satisfies the equation

$$
F(\sigma)^{-1} N F(\sigma)=\sigma N
$$

Hence

Lemma 4.1 A necessary condition for $C$ to be consistent with arbitrary 
$\sigma>0$ is that there exists some matrix $F(\sigma)$ which satisfies (4.4) and which is similar to the direct $r$ th power $\otimes^{r} k(\sigma)^{-1}$ of a non-singular matrix $k(\sigma)^{-1}$.

We shall prove Theorem 2.9 by first finding the characteristic roots of the matrix $F(\sigma)$ satisfying (4.4) and then comparing them with those of the direct $r$ th power $\otimes^{r} k(\sigma)^{-1}$.

Since any $C$ satisfying (4.1) is nilpotent (cf. $\$ 2.6$ ), the Jordan canonical form $N$ of $C$ is of the form

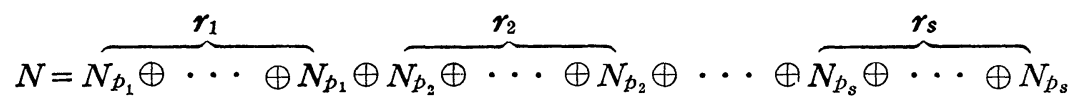

where $p_{1}<p_{2}<\cdots<p_{s}, r_{1} p_{1}+r_{2} p_{2}+\cdots+r_{s} p_{s}=n^{r}$, and $N_{p_{u}}(1 \leq \mu, \nu \leq s)$ is the nilpotent matrix of order $p_{\mu}$ and index $p_{\mu}$ in Jordan canonical form. It is easy to see that the index of nilpotence of $N$, and therefore also of $C$, is $p_{s}$.

We now prove

Lemma 4.2. If the matrix $N$ is given by (4.5), the characteristic roots of any $F(\sigma)$ satisfying (4.4) are (all non-zero and) of the form

$$
\begin{aligned}
& \left(u_{1}, \cdots, u_{r_{1}}\right),\left(u_{1}, \cdots, u_{r_{1}}\right) \sigma, \ldots,\left(u_{1}, \cdots, u_{r_{1}}\right) \sigma^{p_{1}-1} \text {; } \\
& \left(v_{1}, \cdots, v_{r_{2}}\right),\left(v_{1}, \cdots, v_{r_{2}}\right) \sigma, \ldots,\left(v_{1}, \cdots, v_{r_{2}}\right) \sigma^{p_{2}-1} \text {; } \\
& \left(w_{1}, \cdots, w_{r_{s}}\right),\left(w_{1}, \cdots, w_{r_{s}}\right)_{\sigma}, \ldots,\left(w_{1}, \cdots, w_{r_{s}}\right) \sigma^{p_{s}-1} .
\end{aligned}
$$

Proof. Following a well-kncwn procedure for finding all the matrices commutative with a given matrix (cf. Gantmacher [5], p. 220), we can easily show that the most general solution of (4.4) for $F(\sigma)$ is

$$
F(\sigma)=\left(F_{\xi \eta}\right) \quad\left(1 \leq \xi, \eta \leq r_{1}+\cdots+r_{s}\right),
$$

where, for $\xi, \eta$ satisfying

$$
\begin{aligned}
& r_{1}+\cdots+r_{\mu-1}<\xi \leq r_{1}+\cdots+r_{\mu}, \\
& r_{1}+\cdots+r_{\nu-1}<\eta \leq r_{1}+\cdots+r_{\nu}
\end{aligned}
$$

$F_{\xi \eta}$ is a $p_{\mu} \times p_{\nu}$ matrix of the following form

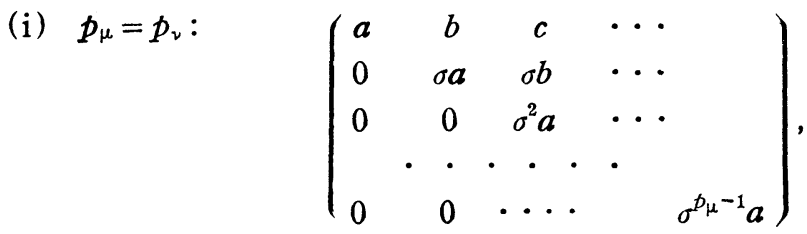




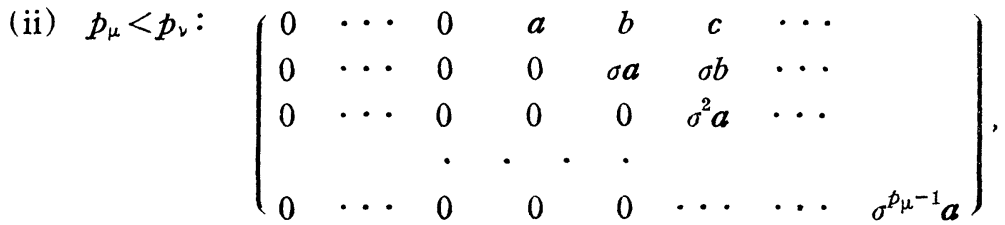

(iii) $p_{\mu}>p_{\nu}$ :

$$
\left(\begin{array}{ccccc}
a & b & c & \cdots & \\
0 & \sigma a & \sigma b & \cdots & \\
0 & 0 & \sigma^{2} a & \cdots & \\
& \cdots & \cdots & & \\
0 & 0 & 0 & \cdots & \sigma^{p_{\nu}-1} a \\
0 & 0 & 0 & \cdots & 0 \\
& \cdots & \cdots & & \\
0 & 0 & 0 & \cdots & 0
\end{array}\right)
$$

where $a, b, c \cdots$ are arbitrary parameters. Now by suitably rearranging the rows and the corresponding columns of $F(\sigma)$, we can obtain a matrix which is similar to $F(\sigma)$ and whose characteristic equation can be found easily. For example, if

$$
N=\left[\begin{array}{cc}
\cdot & 1 \\
\cdot & \cdot
\end{array}\right] \oplus\left[\begin{array}{cc}
\cdot & 1 \\
\cdot & .
\end{array}\right] \oplus\left(\begin{array}{ccc}
\cdot & 1 & \cdot \\
\cdot & \cdot & 1 \\
\cdot & \cdot & \cdot
\end{array}\right),
$$

where and in the next two matrices a dot denotes the zero, then the most general $F(\sigma)$ satisfying $(4.4)$ is

$$
F(\sigma)=\left(\begin{array}{ccccccc}
a_{11} & b_{11} & a_{12} & b_{12} & \cdot & a_{13} & b_{13} \\
\cdot & \sigma a_{11} & \cdot & \sigma a_{12} & \cdot & \cdot & \sigma a_{13} \\
a_{21} & b_{21} & a_{22} & b_{22} & \cdot & a_{23} & b_{23} \\
\cdot & \sigma a_{21} & \cdot & \sigma a_{22} & \cdot & \cdot & \sigma a_{23} \\
a_{31} & b_{31} & a_{32} & b_{32} & a_{33} & \cdot & \cdot \\
\cdot & \sigma a_{31} & \cdot & \sigma a_{32} & \cdot & \sigma a_{33} & \cdot \\
\cdot & \cdot & \cdot & \cdot & \cdot & \cdot & \sigma^{2} a_{33}
\end{array}\right),
$$

and this matrix is similar to

$$
\left(\begin{array}{ccccccc}
a_{11} & a_{12} & \cdot & b_{11} & b_{12} & a_{13} & b_{13} \\
a_{21} & a_{22} & \cdot & b_{21} & b_{22} & a_{23} & b_{23} \\
a_{31} & a_{32} & a_{33} & b_{31} & b_{32} & \cdot & \cdot \\
\cdot & \cdot & \cdot & \sigma a_{11} & \sigma a_{12} & \cdot & \sigma a_{13} \\
\cdot & \cdot & \cdot & \sigma a_{21} & \sigma a_{22} & \cdot & \sigma a_{23} \\
\cdot & \cdot & \cdot & \sigma a_{31} & \sigma a_{32} & \sigma a_{33} & \cdot \\
\cdot & \cdot & \cdot & \cdot & \cdot & \cdot & \sigma^{2} a_{33}
\end{array}\right) \cdot
$$


This shows that the characteristic roots of $F(\sigma)$ are equal to those of the matrices

$$
\left[\begin{array}{ll}
a_{11} & a_{12} \\
a_{21} & a_{22}
\end{array}\right], \quad\left[\begin{array}{ll}
\sigma a_{11} & \sigma a_{12} \\
\sigma a_{21} & \sigma a_{22}
\end{array}\right], \quad\left[a_{33}\right], \quad\left[\sigma a_{33}\right], \quad\left[\sigma^{2} a_{33}\right]
$$

and are therefore of the form

$$
\left(u_{1}, u_{2}\right),\left(u_{1}, u_{2}\right) \sigma ; v_{1}, v_{1} \sigma, v_{1} \sigma^{2}
$$

The general case is similar.

\subsection{The proof}

First, if $\theta_{1}, \ldots, \theta_{n}$ are the characteristic roots of $k(\sigma)^{-1}$, the characteristic roots of the direct $r$ th power $\otimes^{r} k(\sigma)^{-1}$ are the terms in the expansion of $\left(\theta_{1}+\cdots\right.$ $\left.+\theta_{n}\right)^{r}$ (cf. MacDuffee [8] p. 84). Therefore, since $H_{r}^{n}=(n+r-1) ! /(n-1) ! r$ ! is the largest possible number of distinct terms in the expansion of $\left(\theta_{1}+\cdots+\theta_{n}\right)^{r}$, the matrix $\otimes^{r} k(\sigma)^{-1}$ has at most $H_{r}^{n}$ distinct characteristic roots.

On the other hand, since $\sigma>0$ and $\neq 1$, it follows from Lemma 4.2 that any $F(\sigma)$ satisfying (4.4) has at least $p_{s}$ distinct characteristic roots. Now by Lemma 4.1, $F(\sigma)$ and $\otimes^{r} k(\sigma)^{-1}$ have the same characteristic roots. Therefore, we have $p_{s} \leq H_{r}^{n}$. But $p_{s}$ is the index of nilpotence of $C$. Hence Theorem 2.10 (i) is proved.

Next, we know (Chrystal [2], pp. 16-17) that the largest coefficient in the expansion of $\left(\theta_{1}+\cdots+\theta_{n}\right)^{r}$ is

$$
M_{r}^{n}=r ! /(q !)^{n}(q+1)^{p} \quad(r=n q+p, 0 \leq p<n) .
$$

Therefore, at least $M_{r}^{n}$ of the characteristic roots of $\otimes^{r} k(\sigma)^{-1}$ are equal. By Lemma 4.1, the same must be true of $F(\sigma)$. By Lemma 4.2, these $M_{r}^{n}$ equal characteristic roots of $F(\sigma)$ must be distributed one in each of the $r_{1}+\cdots+r_{s}$ different sets

$$
\left(u_{1}, u_{1} \sigma, \cdots, u_{1} \sigma^{p_{1}-1}\right), \ldots,\left(w_{r_{s}}, w_{r_{s}} \sigma, \cdots, w_{r_{s}} \sigma^{p_{s}-1}\right)
$$

because no two characteristic roots in the same set are equal. Therefore, we must have $M_{r}^{n} \leq r_{1}+\cdots+r_{s}$. But $r_{1}+\cdots+r_{s}$ is equal to the number of elementary divisors of the matrix $N$ given by (4.5) and consequently also of C. Hence Theorem 2.10 (ii) is proved.

Remark. Better results than Theorem 2.10 would have been obtained if 
we could compute the elementary divisors of the matrices $F(\sigma)$ and $\otimes^{r} k(\sigma)^{-1}$ and compare them instead of the characterstic roots. Since $\otimes^{r} k(\sigma)^{-1}$ is the direct rth power of $k(\sigma)^{-1}$, its elementary divisors can be explicitly expressed in terms of those of $k(\sigma)^{-1}$ (Roth [10]). But unfortunately, there appears to be no simple way to compute the elementary divisors of $F(\sigma)$; already the elementary divisors of

$$
\left(\begin{array}{cccc}
a & b & c & \cdots \\
0 & a & b & \cdots \\
0 & 0 & a & \cdots \\
& \cdot & \cdot & \cdot \\
0 & 0 & \cdots & a
\end{array}\right)
$$

are rather complicated (cf. Gantmacher [5], p. 156).

\section{REFERENCES}

[1] C. Chevalley, Theory of Lie groups I, Princeton University Press (1946).

[2] G. Chrystal, Algebra II, London (1926).

[3] L. P. Eisenhart, Non-Riemannian geometry, American Math. Soc. (1927).

[4] V. Fukami, Affine connections in almost product manifolds with some structures, Tohoku Math. J., vol. 11 (1959), pp. 430-446.

[5] F. R. Gantmacher, The theory of matrices I, New York, Chelsea (1959).

[6] N. Jacobson, Lectures in abstract algebra II, New York, Van Nostrand (1953).

[7] M. Kurita, "Tensor fields and their parallelisim", Nagoya Math. J., vol. 18 (1961), pp. 133-151.

[8] C. C. MacDuffee, The theory of matrices, New York, Chelsea (1946).

[9] K. Nomizu, Lie groups and differential geometry, Tokyo, Japanese Math. Soc. (1956).

[10] W. E. Roth, "On direct product matrices", Bulletin American Math. Soc., vol. 40 (1964), pp. 461-468.

[11] A. G. Walker, "On parallel fields of partially null vector spaces", Quarterly J. Math. (Oxford), vol. 20 (1949), pp. 135-145.

[12] T. J. Willmore, An introduction to differential geometry, Oxford University Press (1959).

[13] Yung-Chow Wong, "Recurrent tensors on a linearly connected differentiable manifold", Trans. American Math. Soc., vol. 99 (1961), pp. 325-341.

[14] Yung-Chow Wong, "Linear connections with zero torsion and recurrent curvature", Trans. American Math. Soc., vol. 102 (1962), pp. 471-506.

University of Hong Kong,

Hong Kong. 